\title{
Density of Lipschitz functions and equivalence of weak gradients in metric measure spaces
}

\author{
Luigi Ambrosio, Nicola Gigli and Giuseppe Savaré
}

\begin{abstract}
We compare several notions of weak (modulus of) gradients in metric measure spaces and prove their equivalence. Using tools from optimal transportation theory we prove density in energy of Lipschitz maps independently of doubling and Poincaré assumptions on the metric measure space.
\end{abstract}

\section{Introduction}

In the last few years much attention has been devoted to the theory of Sobolev spaces $W^{1, q}$ on metric measure spaces $(X, \mathrm{~d}, \mathfrak{m})$; see for instance [12] and [11] for an overview of this subject. These definitions of Sobolev spaces usually come with a weak definition of the modulus of the gradient, in particular the notion of $q$ upper gradient has been introduced in [16] and used in [20] for a Sobolev space theory. Also, in [20] the notion of minimal $q$-upper gradient has been proved to be equivalent to the notion of relaxed upper gradient arising in Cheeger's paper [6].

In this paper we consider a notion of a gradient $|\nabla f|_{*, q}$, stronger than the one in [6], because in the approximation procedure we use Lipschitz functions and their slopes as upper gradients, and a notion of a $q$-weak upper gradient $|\nabla f|_{w, q}$ weaker than the one of [20], and prove their equivalence. As a consequence all four notions of gradient turn out to be equivalent. A byproduct of our equivalence result is the following density in energy of Lipschitz functions: if $f \in L^{q}(X, \mathfrak{m})$ has a $q$-weak upper gradient $|\nabla f|_{w, q}$ in $L^{q}(X, \mathfrak{m})$, then there exist Lipschitz functions $f_{n}$ converging to $f$ in $L^{q}(X, \mathfrak{m})$ satisfying (here $\left|\nabla f_{n}\right|$ is the slope of $f_{n}$ )

$$
\left.\left.\lim _{n \rightarrow \infty} \int_{X}|| \nabla f_{n}|-| \nabla f\right|_{w, q}\right|^{q} \mathrm{~d} \mathfrak{m}=0
$$

Notice that we can use Mazur's lemma to improve this convergence to strong convergence in $W^{1, q}(X, \mathrm{~d}, \mathfrak{m})$, as soon as this space is reflexive; this happens for 
instance in the context of the spaces with Riemannian Ricci bounds from below considered in [3], with $q=2$.

We emphasize that our density result does not depend on doubling and Poincaré assumptions on the metric measure structure; as is well known (see Theorems 4.14 and 4.24 in [6]), these assumptions ensure the density in Sobolev norm of Lipschitz functions, even in the Lusin sense (i.e. the Lipschitz approximating functions $f_{n}$ coincide with $f$ on larger and larger sets). On the other hand, the density in energy (1.1) suffices for many purposes, for instance the extension by approximation, from Lipschitz to Sobolev functions, of functional inequalities like the Poincaré or Sobolev inequality. For instance, our result can be used to show that if $(X, \mathrm{~d})$ is complete and separable and $\mathfrak{m}$ is a Borel measure finite on bounded sets, then the Poincaré inequality

$$
\int_{B_{r}(x)}\left|f(y)-f_{B_{r}(x)}\right| \mathrm{d} \mathfrak{m}(y) \leq C r \int_{B_{\lambda r}(x)}|\nabla f|(y) \mathrm{d} \mathfrak{m}(y)
$$

holds for all $f: X \rightarrow \mathbb{R}$ Lipschitz on bounded sets if and only if it holds in the form

$$
\int_{B_{r}(x)}\left|f(y)-f_{B_{r}(x)}\right| \mathrm{d} \mathfrak{m}(y) \leq C r \int_{B_{\lambda r}(x)} g(y) \mathrm{d} \mathfrak{m}(y)
$$

for all pairs $(f, g)$ with $f$ Borel and $g$ upper gradient of $f$. This equivalence was proven in [14] for proper, quasiconvex and doubling metric measure spaces, while in [15] (choosing $X=\mathbb{R}^{n} \backslash E$ for suitable compact sets $E$ ) it is proven that completeness of the space cannot be dropped.

The equivalence of these new notions of gradient has been proved in [2] in the case $q=2$; see Corollary 6.3 therein. Here we extend the result to general exponents $q \in(1, \infty)$ and we give a presentation more focused on the equivalence problem. While the traditional proof of density of Lipschitz functions relies on the Poincaré inequality, maximal functions and covering arguments to construct the "optimal" approximating Lipschitz functions $f_{n}$, our proof is more indirect and provides the approximating functions using the $L^{2}$-gradient flow of $\mathrm{Ch}_{q}(f):=$ $q^{-1} \int_{X}|\nabla f|_{*, q}^{q} \mathrm{dm}$ and the analysis of the dissipation rate along this flow of a suitable "entropy" $\int \Phi_{q}(f) \mathrm{dm}$ (in the case $q=2, \Phi(z)=z \log z$ ). This way we prove that $|\nabla f|_{w, q}=|\nabla f|_{*, q}$ m-a.e., and then (1.1) follows by a general property of the minimal $q$-relaxed slope $|\nabla f|_{*, q}$, see Proposition 4.3.

The paper is organized as follows. In Section 2 we recall some preliminary facts on absolutely continuous curves and gradient flows. We also introduce the $p$-th Wasserstein distance and the so-called superposition principle, that allows to pass from an "Eulerian" formulation (i.e. in terms of a curve of measures or a curve of probability densities) to a "Lagrangian" one, namely a probability measure in the space of absolutely continuous paths; this will be the only tool from optimal transportation theory used in the paper.

In Section 3 we study the pointwise properties of the Hopf-Lax semigroup

$$
Q_{t} f(x):=\inf _{y \in X} f(y)+\frac{\mathrm{d}^{p}(x, y)}{p t^{p-1}} .
$$


In comparison with Section 3 of [2], dealing with the case $p=2$, we consider for the sake of simplicity only locally compact spaces and finite distances, but the proofs can be modified to deal with more general cases, see also Section 8. The results of this section overlap with those of the forthcoming paper [10] by Gozlan, Roberto and Samson, where the HL semigroup is used in connection with the proof of transport entropy inequalities.

In Section 4 we introduce the four definitions of gradients we consider, namely:

(1) the Cheeger gradient $|\nabla f|_{C, q}$ of [6] arising from the relaxation of upper gradients;

(2) the minimal relaxed slope $|\nabla f|_{*, q}$ of [2] arising from the relaxation of the slope of Lipschitz functions;

(3) the minimal $q$-upper gradient $|\nabla f|_{S, q}$ of [16] and [20], based on the validity of the upper gradient property out of a $\operatorname{Mod}_{q}$-null set of curves;

(4) the minimal $q$-weak upper gradient of [2], based on the validity of the upper gradient property out of a $q$-null set of curves.

While presenting these definitions we will point out natural relations between them, that lead to the chain of inequalities

$$
|\nabla f|_{w, q} \leq|\nabla f|_{S, q} \leq|\nabla f|_{C, q} \leq|\nabla f|_{*, q} \quad \mathfrak{m} \text {-a.e. in } X
$$

with the concepts of [2] at the extreme sides.

Section 5 contains some basically well known properties of weak gradients, namely chain rules and stability under weak convergence. Section 6 contains the basic facts we shall need on the gradient flow of the lower semicontinuous functional $\mathrm{Ch}_{q}$, in particular the entropy dissipation rate

$$
\frac{\mathrm{d}}{\mathrm{d} t} \int_{X} \Phi\left(f_{t}\right) \mathrm{d} \mathfrak{m}=-\int_{X} \Phi^{\prime \prime}\left(f_{t}\right)\left|\nabla f_{t}\right|_{*, q}^{q} \mathrm{~d} \mathfrak{m}
$$

along this gradient flow.

In Section 7 we prove the equivalence of gradients. Starting from a function $f$ with $|\nabla f|_{w, q} \in L^{q}(X, \mathfrak{m})$ we approximate it by the gradient flow of $f_{t}$ of $\mathrm{Ch}_{q}$ starting from $f$ and we use the weak upper gradient property to get

$$
\limsup _{t \downarrow 0} \frac{1}{t} \int_{0}^{t} \int_{X} \frac{\left|\nabla f_{s}\right|_{*, q}^{q}}{f_{s}^{p-1}} \mathrm{~d} \mathfrak{m} \mathrm{d} s \leq \int_{X} \frac{|\nabla f|_{w, q}^{q}}{f^{p-1}} \mathrm{~d} \mathfrak{m}
$$

where $p=q /(q-1)$ is the dual exponent of $q$. Using the stability properties of the relaxed gradients we eventually get $|\nabla f|_{*, q} \leq|\nabla f|_{*, w} \mathfrak{m}$-a.e. in $X$.

Finally, Section 8 discusses some potential extensions of the results of this paper: we indicate how spaces which are not locally compact and measures that are locally finite can be handled. Other extensions probably require a separate investigation, such as the case of Orlicz spaces and the limiting case $q=1$, corresponding to $W^{1,1}$ and $B V$ spaces. In this latter case the lack of reflexivity of $L^{1}(X, \mathfrak{m})$ poses problems even in the definition of the minimal gradients and we discuss this very briefly.

Acknowledgement. The authors thank P. Koskela for useful comments during the preparation of the paper. 


\section{Preliminary notions}

In this section we introduce some notation and recall a few basic facts on absolutely continuous functions, gradient flows of convex functionals and optimal transportation. See also [1] and [22] as general references.

\subsection{Absolutely continuous curves and slopes}

Let $(X, \mathrm{~d})$ be a metric space, $J \subset \mathbb{R}$ a closed interval and $J \ni t \mapsto x_{t} \in X$. We say that $\left(x_{t}\right)$ is absolutely continuous if

$$
\mathrm{d}\left(x_{s}, x_{t}\right) \leq \int_{s}^{t} g(r) \mathrm{d} r \quad \forall s, t \in J, s<t
$$

for some $g \in L^{1}(J)$. It turns out that, if $\left(x_{t}\right)$ is absolutely continuous, there is a minimal function $g$ with this property, called the metric speed, denoted by $\left|\dot{x}_{t}\right|$ and given for a.e. $t \in J$ by

$$
\left|\dot{x}_{t}\right|=\lim _{s \rightarrow t} \frac{\mathrm{d}\left(x_{s}, x_{t}\right)}{|s-t|} .
$$

See Theorem 1.1.2 in [1] for the simple proof.

We will denote by $C([0,1], X)$ the space of continuous curves from $[0,1]$ to $(X, \mathrm{~d})$ endowed with the sup norm. The set $A C^{p}([0,1], X) \subset C([0,1], X)$ consists of all absolutely continuous curves $\gamma$ such that $\int_{0}^{1}\left|\dot{\gamma}_{t}\right|^{p} \mathrm{~d} t<\infty$ : it is the countable union of the sets $\left\{\gamma: \int_{0}^{1}\left|\dot{\gamma}_{t}\right|^{p} \mathrm{~d} t \leq n\right\}$, which are easily seen to be closed if $p>1$. Thus $A C^{p}([0,1], X)$ is a Borel subset of $C([0,1], X)$. The evaluation maps $\mathrm{e}_{t}$ : $C([0,1], X) \rightarrow X$ are defined by

$$
\mathrm{e}_{t}(\gamma):=\gamma_{t}
$$

and are clearly continuous.

Given $f: X \rightarrow \mathbb{R}$, we define its slope (also called the local Lipschitz constant) by

$$
|\nabla f|(x):=\varlimsup_{y \rightarrow x} \frac{|f(y)-f(x)|}{\mathrm{d}(y, x)} .
$$

For $f, g: X \rightarrow \mathbb{R}$ Lipschitz there clearly holds

$$
\begin{aligned}
|\nabla(\alpha f+\beta g)| & \leq|\alpha||\nabla f|+|\beta||\nabla g| \quad \forall \alpha, \beta \in \mathbb{R}, \\
|\nabla(f g)| & \leq|f||\nabla g|+|g||\nabla f| .
\end{aligned}
$$

We shall also need the following calculus lemma.

Lemma 2.1. Let $f:(0,1) \rightarrow \mathbb{R}, q \in[1, \infty]$, and nonnegative $g \in L^{q}(0,1)$ satisfy

$$
|f(s)-f(t)| \leq\left|\int_{s}^{t} g(r) \mathrm{d} r\right| \quad \text { for } \mathscr{L}^{2} \text {-a.e. }(s, t) \in(0,1)^{2} .
$$

Then $f \in W^{1, q}(0,1)$ and $\left|f^{\prime}\right| \leq g$ a.e. in $(0,1)$. 
Proof. Let $N \subset(0,1)^{2}$ be the $\mathscr{L}^{2}$-negligible subset where the above inequality fails. Choosing $s \in(0,1)$, whose existence is ensured by Fubini's theorem, such that $(s, t) \notin N$ for a.e. $t \in(0,1)$, we obtain that $f \in L^{\infty}(0,1)$. Since the set $\left\{(t, h) \in(0,1)^{2}:(t, t+h) \in N \cap(0,1)^{2}\right\}$ is $\mathscr{L}^{2}$-negligible as well, we can apply Fubini's theorem to obtain that for a.e. $h$ there holds $(t, t+h) \notin N$ for a.e. $t \in(0,1)$. Let $h_{i} \downarrow 0$ with this property and use the identity

$$
\int_{0}^{1} f(t) \frac{\phi(t+h)-\phi(t)}{h} \mathrm{~d} t=-\int_{0}^{1} \frac{f(t-h)-f(t)}{-h} \phi(t) \mathrm{d} t
$$

with $\phi \in C_{c}^{1}(0,1)$ and $h=h_{i}$ sufficiently small to get

$$
\left|\int_{0}^{1} f(t) \phi^{\prime}(t) \mathrm{d} t\right| \leq \int_{0}^{1} g(t)|\phi(t)| \mathrm{d} t
$$

It follows that the distributional derivative of $f$ is a signed measure $\eta$ with finite total variation which satisfies

$$
-\int_{0}^{1} f \phi^{\prime} \mathrm{d} t=\int_{0}^{1} \phi \mathrm{d} \eta, \quad\left|\int_{0}^{1} \phi \mathrm{d} \eta\right| \leq \int_{0}^{1} g|\phi| \mathrm{d} t \quad \text { for every } \phi \in C_{c}^{1}(0,1) .
$$

Therefore $\eta$ is absolutely continuous with respect to Lebesgue measure, with $|\eta| \leq$ $g \mathscr{L}^{1}$. This gives the $W^{1,1}(0,1)$ regularity and, at the same time, the inequality $\left|f^{\prime}\right| \leq g$ a.e. in $(0,1)$. The case $q>1$ follows immediately by applying this inequality when $g \in L^{q}(0,1)$.

\subsection{Gradient flows of convex functionals}

Let $H$ be a Hilbert space, let $\Psi: H \rightarrow \mathbb{R} \cup\{+\infty\}$ be convex and lower semicontinuous and let $D(\Psi)=\{\Psi<\infty\}$ be its finiteness domain. Recall that a gradient flow $x:(0, \infty) \rightarrow H$ of $\Psi$ is a locally absolutely continuous map with values in $D(\Psi)$ satisfying

$$
-\frac{\mathrm{d}}{\mathrm{d} t} x_{t} \in \partial^{-} \Psi\left(x_{t}\right) \quad \text { for a.e. } t \in(0, \infty) .
$$

Here $\partial^{-} \Psi(x)$ is the subdifferential of $\Psi$, defined at any $x \in D(\Psi)$ by

$$
\partial^{-} \Psi(x):=\left\{p \in H^{*}: \Psi(y) \geq \Psi(x)+\langle p, y-x\rangle \forall y \in H\right\} .
$$

We shall use the fact that for all $x_{0} \in \overline{D(\Psi)}$ there exists a unique gradient flow $x_{t}$ of $\Psi$ starting from $x_{0}$, i.e., $x_{t} \rightarrow x_{0}$ as $t \downarrow 0$, and that $t \mapsto \Psi\left(x_{t}\right)$ is nonincreasing and locally absolutely continuous in $(0, \infty)$. In addition, this unique solution exhibits a regularizing effect, namely $-\mathrm{d} / \mathrm{d} t x_{t}$ is the element of minimal norm in $\partial^{-} \Psi\left(x_{t}\right)$ for a.e. $t \in(0, \infty)$.

\subsection{The space $\left(\mathscr{P}(X), W_{p}\right)$ and the superposition principle}

Let $(X, \mathrm{~d})$ be a complete and separable metric space and let $p \in[1, \infty)$. We write $\mathscr{P}(X)$ for the set of all Borel probability measures on $X$. Given $\mu, \nu \in \mathscr{P}(X)$, we 
define the Wasserstein (extended) distance $W_{p}(\mu, \nu) \in[0, \infty]$ between them as

$$
W_{p}^{p}(\mu, \nu):=\min \int \mathrm{d}^{p}(x, y) \mathrm{d} \gamma(x, y)
$$

Here the minimization is made in the class $\Gamma(\mu, \nu)$ of all probability measures $\gamma$ on $X \times X$ such that $\pi_{\#}^{1} \gamma=\mu$ and $\pi_{\#}^{2} \gamma=\nu$, where $\pi^{i}: X \times X \rightarrow X, i=1,2$, are the coordinate projections and $f_{\#}: \mathscr{P}(Y) \rightarrow \mathscr{P}(Z)$ is the push-forward operator induced by a Borel map $f: Y \rightarrow Z$.

An equivalent definition of $W_{p}$ comes from the dual formulation of the transport problem. In the case when $(X, \mathrm{~d})$ has finite diameter the dual formulation takes the simplified form

$$
\frac{1}{p} W_{p}^{p}(\mu, \nu)=\sup _{\psi \in \operatorname{Lip}(X)} \int \psi \mathrm{d} \mu+\int \psi^{c} \mathrm{~d} \nu,
$$

where the $c$-transform $\psi^{c}$ is defined by

$$
\psi^{c}(y):=\inf _{x \in X} \frac{\mathrm{d}^{p}(x, y)}{p}-\psi(x) .
$$

We will need the following result, proved in [18]. It shows how to associate to a curve $\mu_{t}$ absolutely continuous with respecto to $W_{p}$ a plan $\pi \in \mathscr{P}(C([0,1], X))$ representing the curve itself (see also Theorem 8.2.1 in [1] for the Euclidean case).

Proposition 2.2 (Superposition principle). Let $(X, \mathrm{~d})$ be a complete and separable metric space with $\mathrm{d}$ bounded, $p \in(1, \infty)$ and let $\mu_{t} \in A C^{p}\left([0, T] ;\left(\mathscr{P}(X), W_{p}\right)\right)$. Then there exists $\pi \in \mathscr{P}(C([0,1], X))$, concentrated on $A C^{p}([0,1], X)$, such that $\left(\mathrm{e}_{t}\right)_{\sharp} \boldsymbol{\pi}=\mu_{t}$ for any $t \in[0, T]$ and

$$
\int\left|\dot{\gamma}_{t}\right|^{p} \mathrm{~d} \boldsymbol{\pi}(\gamma)=\left|\dot{\mu}_{t}\right|^{p} \quad \text { for a.e. } t \in[0, T] .
$$

\section{Hopf-Lax formula and Hamilton-Jacobi equation}

The aim of this section is to study the properties of the Hopf-Lax formula in a metric setting and its relations with the Hamilton-Jacobi equation. Here we assume for simplicity that $(X, \mathrm{~d})$ is a compact metric space, see Section 8 for a more general discussion. Notice that there is no reference measure $\mathfrak{m}$ here. We fix a power $p \in(1, \infty)$ and denote by $q$ the dual exponent.

Let $f: X \rightarrow \mathbb{R}$ be a Lipschitz function. For $t>0$ define

$$
F(t, x, y):=f(y)+\frac{\mathrm{d}^{p}(x, y)}{p t^{p-1}},
$$

and the function $Q_{t} f: X \rightarrow \mathbb{R}$ by

$$
Q_{t} f(x):=\inf _{y \in X} F(t, x, y)=\min _{y \in X} F(t, x, y) .
$$


Also, we introduce the functions $D^{+}, D^{-}: X \times(0, \infty) \rightarrow \mathbb{R}$ defined by

$$
\begin{aligned}
& D^{+}(x, t):=\max \mathrm{d}(x, y), \\
& D^{-}(x, t):=\min \mathrm{d}(x, y),
\end{aligned}
$$

where, in both cases, the $y$ 's vary over all minima of $F(t, x, \cdot)$. We also set $Q_{0} f=f$ and $D^{ \pm}(x, 0)=0$. Arguing as in Lemma 3.1.2 of [1] it is easy to check that the map $[0, \infty) \ni(t, x) \mapsto Q_{t} f(x)$ is continuous. Furthermore, the fact that $f$ is Lipschitz yields easily

$$
D^{-}(x, t) \leq D^{+}(x, t) \leq t(p \operatorname{Lip}(f))^{1 /(p-1)} .
$$

Proposition 3.1 (Monotonicity of $D^{ \pm}$). For all $x \in X$ there holds

$$
D^{+}(x, t) \leq D^{-}(x, s) \quad 0 \leq t<s .
$$

As a consequence, $D^{+}(x, \cdot)$ and $D^{-}(x, \cdot)$ are both nondecreasing, and they coincide, with at most countably many exceptions, in $[0, \infty)$.

Proof. Fix $x \in X$. For $t=0$ there is nothing to prove. Now pick $0<t<s$ and choose minimizers $x_{t}$ and $x_{s}$ of $F(t, x, \cdot)$ and $F(s, x, \cdot)$ respectively, such that $\mathrm{d}\left(x, x_{t}\right)=D^{+}(x, t)$ and $\mathrm{d}\left(x, x_{s}\right)=D^{-}(x, s)$. The minimality of $x_{t}$ and $x_{s}$ gives

$$
\begin{aligned}
& f\left(x_{t}\right)+\frac{\mathrm{d}^{p}\left(x_{t}, x\right)}{p t^{p-1}} \leq f\left(x_{s}\right)+\frac{\mathrm{d}^{p}\left(x_{s}, x\right)}{p t^{p-1}} \\
& f\left(x_{s}\right)+\frac{\mathrm{d}^{p}\left(x_{s}, x\right)}{p s^{p-1}} \leq f\left(x_{t}\right)+\frac{\mathrm{d}^{p}\left(x_{t}, x\right)}{p s^{p-1}} .
\end{aligned}
$$

Summing these and using the fact that $1 / t \geq 1 / s$, we deduce

$$
D^{+}(x, t)=\mathrm{d}\left(x_{t}, x\right) \leq \mathrm{d}\left(x_{s}, x\right)=D^{-}(x, s),
$$

which is (3.5). Combining this with the inequality $D^{-} \leq D^{+}$we immediately obtain that both functions are nonincreasing. At a point of right continuity of $D^{-}(x, \cdot)$ we get

$$
D^{+}(x, t) \leq \inf _{s>t} D^{-}(x, s)=D^{-}(x, t)
$$

This implies that the two functions coincide out of a countable set.

Next, we examine the semicontinuity properties of $D^{ \pm}$. These properties imply that points $(x, t)$ where the equality $D^{+}(x, t)=D^{-}(x, t)$ occurs are continuity points for both $D^{+}$and $D^{-}$.

Proposition 3.2 (Semicontinuity of $D^{ \pm}$). $D^{+}$is upper semicontinuous and $D^{-}$ is lower semicontinuous in $X \times[0, \infty)$. 
Proof. We prove lower semicontinuity of $D^{-}$, the proof of upper semicontinuity of $D^{+}$being similar. Let $\left(x_{i}, t_{i}\right)$ be any sequence converging to $(x, t)$ such that the limit of $D^{-}\left(x_{i}, t_{i}\right)$ exists and assume that $t>0$ (the case $t=0$ is trivial). For every $i$, let $\left(y_{i}\right)$ be a minimum of $F\left(t_{i}, x_{i}, \cdot\right)$ for which $\mathrm{d}\left(y_{i}, x_{i}\right)=D^{-}\left(x_{i}, t_{i}\right)$, so that

$$
f\left(y_{i}\right)+\frac{\mathrm{d}^{p}\left(y_{i}, x_{i}\right)}{p t_{i}^{p-1}}=Q_{t_{i}} f\left(x_{i}\right) .
$$

The continuity of $(x, t) \mapsto Q_{t} f(x)$ gives that $\lim _{i} Q_{t_{i}} f\left(x_{i}\right)=Q_{t} f(x)$, thus

$$
\lim _{i \rightarrow \infty} f\left(y_{i}\right)+\frac{\mathrm{d}^{p}\left(y_{i}, x\right)}{p t^{p-1}}=Q_{t} f(x) .
$$

That is, $i \mapsto y_{i}$ is a minimizing sequence for $F(t, x, \cdot)$. Since $(X, \mathrm{~d})$ is compact, possibly passing to a subsequence, not relabeled, we may assume that $\left(y_{i}\right)$ converges to some $y$ as $i \rightarrow \infty$. Therefore

$$
D^{-}(x, t) \leq \mathrm{d}(x, y)=\lim _{i \rightarrow \infty} \mathrm{d}\left(x_{i}, y_{i}\right)=\lim _{i \rightarrow \infty} D^{-}\left(x_{i}, t_{i}\right) .
$$

Proposition 3.3 (Time derivative of $Q_{t} f$ ). The map $t \mapsto Q_{t} f$ is Lipschitz from $[0, \infty)$ to $C(X)$ and, for all $x \in X$, it satisfies:

$$
\frac{\mathrm{d}}{\mathrm{d} t} Q_{t} f(x)=-\frac{1}{q}\left[\frac{D^{ \pm}(x, t)}{t}\right]^{p},
$$

for any $t>0$, with at most countably many exceptions.

Proof. Let $t<s$ and let $x_{t}$ and $x_{s}$ be minima of $F(t, x, \cdot)$ and $F(s, x, \cdot)$. We have

$$
\begin{aligned}
& Q_{s} f(x)-Q_{t} f(x) \leq F\left(s, x, x_{t}\right)-F\left(t, x, x_{t}\right)=\frac{\mathrm{d}^{p}\left(x, x_{t}\right)}{p} \frac{t^{p-1}-s^{p-1}}{t^{p-1} s^{p-1}}, \\
& Q_{s} f(x)-Q_{t} f(x) \geq F\left(s, x, x_{s}\right)-F\left(t, x, x_{s}\right)=\frac{\mathrm{d}^{p}\left(x, x_{s}\right)}{p} \frac{t^{p-1}-s^{p-1}}{t^{p-1} s^{p-1}},
\end{aligned}
$$

which gives that $t \mapsto Q_{t} f(x)$ is Lipschitz in $(\varepsilon, \infty)$ for any $\varepsilon>0$ uniformly with respect to $x \in X$. Also, dividing by $(s-t)$ and taking Proposition 3.1 into account, we get (3.6). Now notice that from (3.4) we get that $q\left|\frac{\mathrm{d}}{\mathrm{d} t} Q_{t} f(x)\right| \leq p^{q}[\operatorname{Lip}(f)]^{q}$ for any $x \in X$ and a.e. $t$, which, together with the pointwise convergence of $Q_{t} f$ to $f$ as $t \downarrow 0$, yields that $t \mapsto Q_{t} f \in C(X)$ is Lipschitz in $[0, \infty)$.

In the next proposition we bound the slope of the function $Q_{t} f$ at $x$ from above by $\left|D^{+}(x, t) / t\right|^{p-1}$; actually we shall prove a more precise statement, in connection with $\S 8.3$, which involves the asymptotic Lipschitz constant

$$
\operatorname{Lip}_{a}(f, x):=\inf _{r>0} \operatorname{Lip}\left(f, B_{r}(x)\right)=\lim _{r \downarrow 0} \operatorname{Lip}\left(f, B_{r}(x)\right) .
$$

Notice that $\operatorname{Lip}(f) \geq \operatorname{Lip}_{a}(f, x) \geq|\nabla f|^{*}(x)$, where $|\nabla f|^{*}$ is the upper semicontinuous envelope of the slope of $f$. The second inequality is easily seen to be an equality in length spaces. 
Proposition 3.4 (Bound on the asymptotic Lipschitz constant of $Q_{t} f$ ). For $(x, t) \in X \times(0, \infty)$ there holds:

$$
\operatorname{Lip}_{a}\left(Q_{t} f, x\right) \leq\left[\frac{D^{+}(x, t)}{t}\right]^{p-1} .
$$

In particular $\operatorname{Lip}\left(Q_{t}(f)\right) \leq p \operatorname{Lip}(f)$.

Proof. Fix $y, z \in X, t \in(0, \infty)$ and a minimizer $\bar{y}$ for $F(t, y, \cdot)$. Since there holds

$$
\begin{aligned}
Q_{t} f(z)-Q_{t} f(y) & \leq F(t, z, \bar{y})-F(t, y, \bar{y})=f(\bar{y})+\frac{\mathrm{d}^{p}(z, \bar{y})}{p t^{p-1}}-f(\bar{y})-\frac{\mathrm{d}^{p}(y, \bar{y})}{p t^{p-1}} \\
& \leq \frac{(\mathrm{d}(z, y)+\mathrm{d}(y, \bar{y}))^{p}}{p t^{p-1}}-\frac{\mathrm{d}^{p}\left(x_{i}, y_{i}\right)}{p t^{p-1}} \\
& \leq \frac{\mathrm{d}(z, y)}{t^{p-1}}\left(\mathrm{~d}(z, y)+D^{+}(y, t)\right)^{p-1},
\end{aligned}
$$

dividing by $\mathrm{d}(z, y)$ and inverting the roles of $y$ and $z$ gives

$$
\operatorname{Lip}\left(Q_{t} f, B_{r}(x)\right) \leq t^{1-p}\left(\sup _{y \in B_{r}(x)} D^{+}(y, t)\right)^{p-1} .
$$

Letting $r \downarrow 0$ and using the upper semicontinuity of $D^{+}$we get (3.8).

Finally, the bound on the Lipschitz constant of $Q_{t} f$ follows directly from (3.4) and (3.8).

Theorem 3.5 (Subsolution of HJ). For every $x \in X$ there holds

$$
\frac{\mathrm{d}}{\mathrm{d} t} Q_{t} f(x)+\frac{1}{q}\left|\nabla Q_{t} f\right|^{q}(x) \leq 0
$$

for every $t \in(0, \infty)$, with at most countably many exceptions.

Proof. The claim is a direct consequence of Propositions 3.3 and 3.4.

Notice also that (3.8) facilitates writing the HJ subsolution property in a stronger form using the asymptotic Lipschitz constant $\operatorname{Lip}_{a}\left(Q_{t} f, \cdot\right)$ in place of $\left|\nabla Q_{t} f\right|$. Namely, for all $x \in X$ there holds

$$
\frac{\mathrm{d}}{\mathrm{d} t} Q_{t} f(x)+\frac{1}{q}\left(\operatorname{Lip}_{a}\left(Q_{t} f, x\right)\right)^{q} \leq 0
$$

for every $t \in(0, \infty)$, with at most countably many exceptions.

We just proved that in an arbitrary metric space the Hopf-Lax formula produces subsolutions of the Hamilton-Jacobi equations. In geodesic spaces this result can be improved to get solutions. Since we shall not need the result, we just state it (the proof is analogous to that of Proposition 3.6 in [2]).

Theorem 3.6 (Supersolution of HJ). Assume that $(X, \mathrm{~d})$ is a geodesic space. Then equality holds in (3.8). In particular, for all $x \in X$ there holds

$$
\frac{\mathrm{d}}{\mathrm{d} t} Q_{t} f(x)+\frac{1}{q}\left|\nabla Q_{t} f\right|^{q}(x)=0
$$

for every $t \in(0, \infty)$, with at most countably many exceptions. 


\section{Weak gradients}

Let $(X, \mathrm{~d})$ be a complete and separable metric space and let $\mathfrak{m}$ be a nonnegative $\sigma$-finite Borel measure in $X$. In this section we introduce and compare four notions of weak gradients, the gradient $|\nabla f|_{C, q}$ introduced in [6], the gradient $|\nabla f|_{S, q}$ introduced in [16] and further studied in [20] and the gradients $|\nabla f|_{*, q}$ and $|\nabla f|_{w, q}$ whose definition can be obtained adapting to general power functions the approach of [2]. We will also see that

$$
|\nabla f|_{w, q} \leq|\nabla f|_{S, q} \leq|\nabla f|_{C, q} \leq|\nabla f|_{*, q} \quad \text { m-a.e. in } X \text {. }
$$

We shall prove in Section 7 that actually all inequalities are equalities, by proving equality of the two extremes. As in the previous section, we shall denote by $p$ the dual exponent of $q$.

\subsection{Upper gradients}

Following [13], we say that a Borel function $g$ is an upper gradient of a Borel function $f: X \rightarrow \mathbb{R}$ if the inequality

$$
\left|\int_{\partial \gamma} f\right| \leq \int_{\gamma} g
$$

holds for all absolutely continuous curves $\gamma:[0,1] \rightarrow X$. Here $\int_{\partial \gamma} f=f\left(\gamma_{1}\right)-f\left(\gamma_{0}\right)$, while $\int_{\gamma} g=\int_{0}^{1} g\left(\gamma_{s}\right)\left|\dot{\gamma}_{s}\right| \mathrm{d} s$.

It is well known and easy to check that the slope is an upper gradient, for locally Lipschitz functions.

\subsection{Cheeger's gradient $|\nabla f|_{C, q}$}

The following definition is taken from [6], where weak gradients are defined from upper gradients via a relaxation procedure.

Definition 4.1 ( $q$-relaxed upper gradient). We say that $g \in L^{q}(X, \mathfrak{m})$ is a $q$-relaxed upper gradient of $f \in L^{q}(X, \mathfrak{m})$ if there exist $\tilde{g} \in L^{q}(X, \mathfrak{m})$, functions $f_{n} \in L^{q}(X, \mathfrak{m})$ and an upper gradient $g_{n}$ of $f_{n}$ such that:

(a) $f_{n} \rightarrow f$ in $L^{q}(X, \mathfrak{m})$ and the $g_{n}$ converge weakly to $\tilde{g}$ in $L^{q}(X, \mathfrak{m})$;

(b) $\tilde{g} \leq g$ m-a.e. in $X$.

We say that $g$ is a minimal $q$-relaxed upper gradient of $f$ if its $L^{q}(X, \mathfrak{m})$ norm is minimal among $q$-relaxed upper gradients. We shall denote by $|\nabla f|_{C, q}$ the minimal $q$-relaxed upper gradient.

\subsection{Minimal $q$-relaxed slope $|\nabla f|_{*, q}$}

The second definition of weak gradient we shall consider is a variant of the previous one and arises by relaxing the integral of the $q$-th power of the slope of Lipschitz 
functions. In comparison with Definition 4.1, we are considering only Lipschitz approximating functions and we are taking their slopes as upper gradients. In the spirit of Sobolev space theory, this should be considered as an " $H$ definition", since an approximation with Lipschitz functions is involved.

Definition 4.2 (Relaxed slope). We say that $g \in L^{q}(X, \mathfrak{m})$ is a $q$-relaxed slope of $f \in L^{q}(X, \mathfrak{m})$ if there exist $\tilde{g} \in L^{q}(X, \mathfrak{m})$ and Lipschitz functions $f_{n} \in L^{q}(X, \mathfrak{m})$ such that:

(a) $f_{n} \rightarrow f$ in $L^{q}(X, \mathfrak{m})$ and the $\left|\nabla f_{n}\right|$ converge weakly to $\tilde{g}$ in $L^{q}(X, \mathfrak{m})$;

(b) $\tilde{g} \leq g$ m-a.e. in $X$.

We say that $g$ is the minimal $q$-relaxed slope of $f$ if its $L^{q}(X, \mathfrak{m})$ norm is minimal among $q$-relaxed slopes. We shall denote by $|\nabla f|_{*, q}$ the minimal $q$-relaxed slope.

By this definition and the sequential compactness of weak topologies, any $L^{q}$ limit of Lipschitz functions $f_{n}$ with $\int\left|\nabla f_{n}\right|^{q} \mathrm{dm}$ uniformly bounded has a $q$-relaxed slope. On the other hand, using Mazur's lemma (see Lemma 4.3 in [2] for details), the definition of $q$-relaxed slope would be unchanged if the weak convergence of $\left|\nabla f_{n}\right|$ in (a) were replaced by the condition $\left|\nabla f_{n}\right| \leq g_{n}$ and $g_{n} \rightarrow \tilde{g}$ strongly in $L^{q}(X, \mathfrak{m})$. This alternative characterization of $q$-relaxed slopes is suitable for diagonal arguments and proves, together with (2.1a), that the collection of $q$-relaxed slopes is a closed convex set, possibly empty. Hence, thanks to the uniform convexity of $L^{q}(X, \mathfrak{m})$, the definition of $|\nabla f|_{*, q}$ is well posed. Also, arguing as in [2] and using once more the uniform convexity of $L^{q}(X, \mathfrak{m})$, it is not difficult to show the following result:

Proposition 4.3. If $f \in L^{q}(X, \mathfrak{m})$ has a q-relaxed slope, then there exist Lipschitz functions $f_{n}$ satisfying

$$
\lim _{n \rightarrow \infty} \int_{X}\left|f_{n}-f\right|^{q} \mathrm{~d} \mathfrak{m}+\left.\left.\int_{X}|| \nabla f_{n}|-| \nabla f\right|_{*, q}\right|^{q} \mathrm{~d} \mathfrak{m}=0 .
$$

Since the slope is an upper gradient for Lipschitz functions, any $q$-relaxed slope is a $q$-relaxed upper gradient, hence

$$
|\nabla f|_{C, q} \leq|\nabla f|_{*, q} \quad \mathfrak{m} \text {-a.e. in } X
$$

whenever $f$ has a $q$-relaxed slope.

Remark 4.4. Notice that in principle the integrability of $f$ could be decoupled from the integrability of the gradient, because no global Poincaré inequality can be expected at this level of generality. Indeed, to increase the symmetry with the next two gradients, one might even consider the convergence $\mathfrak{m}$-a.e. of the approximating functions, removing any integrability assumption. We have left the convergence in $L^{q}$ because this presentation is more consistent with the usual presentations of Sobolev spaces, and the definitions given in [6] and [2]. Using locality and a truncation argument, the definitions can be extended to more general classes of functions, see (6.2). 


\section{4. $q$-upper gradients and $|\nabla f|_{S, q}$}

Here we recall a weak definition of upper gradient, taken from [16] and further studied in [20] in connection with the theory of Sobolev spaces, where we allow for exceptions in (4.2). Recall that, for $\Gamma \subset A C([0,1], X)$, the $q$-modulus $\operatorname{Mod}_{q}(\Gamma)$ is defined by (see [8] for a systematic analysis of this concept)

$$
\operatorname{Mod}_{q}(\Gamma):=\inf \left\{\int_{X} \rho^{q} \mathrm{dm}: \int_{\gamma} \rho \geq 1 \quad \forall \gamma \in \Gamma\right\} .
$$

We say that $\Gamma$ is $\operatorname{Mod}_{q}$-negligible if $\operatorname{Mod}_{q}(\Gamma)=0$. Accordingly, we say that a Borel function $g: X \rightarrow[0, \infty]$ is a $q$-upper gradient of $f$ if there exist a function $\tilde{f}$ and a $\operatorname{Mod}_{q}$-negligible set $\Gamma$ such that $\tilde{f}=f \mathfrak{m}$-a.e. in $X$ and

$$
\left|\tilde{f}\left(\gamma_{0}\right)-\tilde{f}\left(\gamma_{1}\right)\right| \leq \int_{\gamma} g, \quad \forall \gamma \in A C([0,1], X) \backslash \Gamma .
$$

It is not hard to prove that the collection of all $q$-upper gradients of $f$ is convex and closed, so that we can call minimal $q$-upper gradient, and denote by $|\nabla f|_{S, q}$, the element with minimal $L^{q}(X, \mathfrak{m})$ norm. Furthermore, the inequality

$$
|\nabla f|_{S, q} \leq|\nabla f|_{C, q} \quad \mathfrak{m} \text {-a.e. in } X
$$

(namely, the fact that all $q$-relaxed upper gradients are $q$-upper gradients) follows by a stability property of $q$-upper gradients very similar to the one stated in Theorem 5.3 below for $q$-weak upper gradients, see Lemma 4.11 in [20]. Finally, an observation due to Fuglede (see Remark 4.5 below) shows that any $q$-upper gradient can be strongly approximated in $L^{q}(X, \mathfrak{m})$ by upper gradients. This has been used in [20] to show that the equality $|\nabla f|_{S, q}=|\nabla f|_{C, q}$ m-a.e. in $X$ holds.

Remark 4.5 (Fuglede). If $\operatorname{Mod}_{q}(\Gamma)=0$ and $\varepsilon>0$, then we can find $\rho \in L^{q}(X, \mathfrak{m})$ with $\|\rho\|_{q}<\varepsilon$ and $\int_{\gamma} \rho=\infty$ for all $\gamma \in \Gamma$. Indeed, if we choose functions $\rho_{n} \in$ $L^{q}(X, \mathfrak{m})$ with $\left\|\rho_{n}\right\|_{q}<1 / n$ and $\int_{\gamma} \rho_{n} \geq 1$ for all $\gamma \in \Gamma$, the function

$$
\rho:=\sum_{n \geq 1} \frac{\delta}{n} \rho_{n}
$$

has the required property for $\delta=\delta(\varepsilon)>0$ small enough.

\section{5. $q$-weak upper gradients and $|\nabla f|_{w, q}$}

Recall that the evaluation maps $\mathrm{e}_{t}: C([0,1], X) \rightarrow X$ are defined by $\mathrm{e}_{t}(\gamma):=\gamma_{t}$. We also introduce the restriction maps $\operatorname{restr}_{t}^{s}: C([0,1], X) \rightarrow C([0,1], X), 0 \leq t \leq$ $s \leq 1$, given by

$$
\operatorname{restr}_{t}^{s}(\gamma)_{r}:=\gamma_{(1-r) t+r s}
$$

so that $\operatorname{restr}_{t}^{s}$ "stretches" the restriction of the curve to $[s, t]$ to the whole of $[0,1]$.

Our definition of $q$-weak upper gradient still allows for exceptions in (4.2), but with a different notion of exceptional set. See also Remark 4.12 below. 
Definition 4.6 (Test plans and negligible sets of curves). We say that a probability measure $\boldsymbol{\pi} \in \mathscr{P}(C([0,1], X))$ is a $p$-test plan if $\boldsymbol{\pi}$ is concentrated on $A C^{p}([0,1], X)$, $\iint_{0}^{1}\left|\dot{\gamma}_{t}\right|^{p} \mathrm{~d} t \mathrm{~d} \boldsymbol{\pi}<\infty$, and there exists a constant $C(\boldsymbol{\pi})$ such that

$$
\left(\mathrm{e}_{t}\right)_{\#} \boldsymbol{\pi} \leq C(\boldsymbol{\pi}) \mathfrak{m} \quad \forall t \in[0,1] .
$$

A Borel set $A \subset C([0,1], X)$ is said to be $q$-negligible if $\pi(A)=0$ for any $p$-test plan $\boldsymbol{\pi}$. A property which holds for every $\gamma \in C([0,1], X)$, except possibly a $q$-negligible set, is said to hold for $q$-almost every curve.

Observe that, by definition, $C([0,1], X) \backslash A C^{p}([0,1], X)$ is $q$-negligible, so the notion starts to be meaningful when we look at subsets $A$ of $A C^{p}([0,1], X)$.

Remark 4.7. An easy consequence of condition (4.8) is that if two m-measurable functions $f, g: X \rightarrow \mathbb{R}$ coincide up to a $\mathfrak{m}$-negligible set and $\mathcal{T}$ is an at most countable subset of $[0,1]$, then the functions $f \circ \gamma$ and $g \circ \gamma$ coincide in $\mathcal{T}$ for $q$-almost every curve $\gamma$.

Moreover, choosing an arbitrary $p$-test plan $\boldsymbol{\pi}$ and applying Fubini's Theorem to the product measure $\mathscr{L}^{1} \times \boldsymbol{\pi}$ in $(0,1) \times C([0,1] ; X)$ we also obtain that $f \circ \gamma=g \circ \gamma$ $\mathscr{L}^{1}$-a.e. in $(0,1)$ for $\pi$-a.e. curve $\gamma$; since $\boldsymbol{\pi}$ is arbitrary, the same property holds for $q$-a.e. $\gamma$.

Coupled with the definition of $q$-negligible set of curves, there are the definitions of $q$-weak upper gradient and of functions which are Sobolev along $q$-a.e. curve.

Definition 4.8 ( $q$-weak upper gradients). A Borel function $g: X \rightarrow[0, \infty]$ is a $q$-weak upper gradient of $f: X \rightarrow \mathbb{R}$ if

$$
\left|\int_{\partial \gamma} f\right| \leq \int_{\gamma} g \text { for } q \text {-a.e. } \gamma \text {. }
$$

Definition 4.9 (Sobolev functions along $q$-a.e. curve). A function $f: X \rightarrow \mathbb{R}$ is Sobolev along $q$-a.e. curve if for $q$-a.e. curve $\gamma$ the function $f \circ \gamma$ coincides a.e. in $[0,1]$ and in $\{0,1\}$ with an absolutely continuous map $f_{\gamma}:[0,1] \rightarrow \mathbb{R}$.

By Remark 4.7 applied to $\mathcal{T}:=\{0,1\},(4.9)$ does not depend on the particular representative of $f$ in the class of $\mathfrak{m}$-measurable function coinciding with $f$ up to an $\mathfrak{m}$-negligible set. The same remark also shows that the property of being Sobolev along $q$-a.e. curve $\gamma$ is independent of the representative in the class of $\mathfrak{m}$-measurable functions coinciding with $f \mathfrak{m}$-a.e. in $X$.

In the next remark, using Lemma 2.1, we prove that the existence of a $q$-weak upper gradient $g$ such that $\int_{\gamma} g<\infty$ for $q$-a.e. $\gamma$ (in particular if $g \in L^{q}(X, \mathfrak{m})$ ) implies Sobolev regularity along $q$-a.e. curve. Notice that we only recently we realized the validity of this implication, compare with the only apparently stronger definitions given in [2]. 
Remark 4.10 (Equivalence with the axiomatization in [2]). Notice that if $\boldsymbol{\pi}$ is a $p$-test plan, so is $\left(\operatorname{restr}_{t}^{s}\right)_{\sharp} \boldsymbol{\pi}$. Hence if $g$ is a $q$-weak upper gradient of $f$ such that $\int_{\gamma} g<\infty$ for $q$-a.e. $\gamma$, then for every $t<s$ in $[0,1]$ there holds

$$
\left|f\left(\gamma_{s}\right)-f\left(\gamma_{t}\right)\right| \leq \int_{t}^{s} g\left(\gamma_{r}\right)\left|\dot{\gamma}_{r}\right| \mathrm{d} r \quad \text { for } q \text {-a.e. } \gamma
$$

Let $\boldsymbol{\pi}$ be a $p$-test plan. By Fubini's theorem applied to the product measure $\mathscr{L}^{2} \times \boldsymbol{\pi}$ in $(0,1)^{2} \times C([0,1] ; X)$, for $\pi$-a.e. $\gamma$ the function $f$ satisfies

$$
\left|f\left(\gamma_{s}\right)-f\left(\gamma_{t}\right)\right| \leq\left|\int_{t}^{s} g\left(\gamma_{r}\right)\right| \dot{\gamma}_{r}|\mathrm{~d} r| \quad \text { for } \mathscr{L}^{2} \text {-a.e. }(t, s) \in(0,1)^{2} .
$$

An analogous argument shows that

$$
\left\{\begin{array}{l}
\left|f\left(\gamma_{s}\right)-f\left(\gamma_{0}\right)\right| \leq \int_{0}^{s} g\left(\gamma_{r}\right)\left|\dot{\gamma}_{r}\right| \mathrm{d} r \\
\left|f\left(\gamma_{1}\right)-f\left(\gamma_{s}\right)\right| \leq \int_{s}^{1} g\left(\gamma_{r}\right)\left|\dot{\gamma}_{r}\right| \mathrm{d} r
\end{array} \quad \text { for } \mathscr{L}^{1} \text {-a.e. } s \in(0,1) .\right.
$$

Since $g \circ \gamma|\dot{\gamma}| \in L^{1}(0,1)$ for $\pi$-a.e. $\gamma$, by Lemma 2.1 it follows that $f \circ \gamma \in W^{1,1}(0,1)$ for $\pi$-a.e. $\gamma$, and

$$
\left|\frac{\mathrm{d}}{\mathrm{d} t}(f \circ \gamma)\right| \leq g \circ \gamma|\dot{\gamma}| \quad \text { a.e. in }(0,1) \text {, for } \pi \text {-a.e. } \gamma \text {. }
$$

Since $\boldsymbol{\pi}$ is arbitrary, we conclude that $f \circ \gamma \in W^{1,1}(0,1)$ for $q$-a.e. $\gamma$, and therefore $f \circ \gamma$ admits an absolutely continuous representative $f_{\gamma}$; moreover, by (4.10), it is immediate to check that $f(\gamma(t))=f_{\gamma}(t)$ for $t \in\{0,1\}$ and $q$-a.e. $\gamma$.

It is immediate to show, using the same argument as in the previous remark that if $f$ is Sobolev along $q$-a.e. curve there holds

$g_{1}, g_{2} q$-weak upper gradients of $f \Longrightarrow \min \left\{g_{1}, g_{2}\right\}$-weak upper gradient of $f$.

Using this stability property we can recover, again, a distinguished minimal object.

Definition 4.11 (Minimal $q$-weak upper gradient). Let $f: X \rightarrow \mathbb{R}$ be Sobolev along $q$-a.e. curve. The minimal $q$-weak upper gradient $|\nabla f|_{w, q}$ of $f$ is the $q$-weak upper gradient characterized, up to $\mathfrak{m}$-negligible sets, by the property

$$
|\nabla f|_{w, q} \leq g \quad \mathfrak{m} \text {-a.e. in } X \text {, for every } q \text {-weak upper gradient } g \text { of } f .
$$

Uniqueness of the minimal weak upper gradient is obvious. For existence, since $\mathfrak{m}$ is $\sigma$-finite we can find a Borel and $\mathfrak{m}$-integrable function $\theta: X \rightarrow(0, \infty)$ and $|\nabla f|_{w, q}:=\inf _{n} g_{n}$, where $g_{n}$ are $q$-weak upper gradients which provide a minimizing sequence in

$$
\inf \left\{\int_{X} \theta \tan ^{-1} g \mathrm{dm}: g \text { is a } q \text {-weak upper gradient of } f\right\} .
$$

We immediately see, thanks to (4.12), that we can assume with no loss of generality that $g_{n+1} \leq g_{n}$. Hence, by monotone convergence, the function $|\nabla f|_{w, q}$ is a $q$-weak upper gradient of $f$ and $\int_{X} \theta \tan ^{-1} g \mathrm{dm}$ is minimal at $g=|\nabla f|_{w} q$. This minimality, in conjunction with (4.12), gives (4.13). 
Remark 4.12. Observe that for a Borel set $\Gamma \subset C([0,1], X)$ and a test plan $\boldsymbol{\pi}$, integrating the inequality $\int_{\gamma} \rho \geq 1$ on $\Gamma$ w.r.t. $\pi$, and then minimizing over $\rho$, we get

$$
\boldsymbol{\pi}(\Gamma) \leq(C(\boldsymbol{\pi}))^{1 / q}\left(\operatorname{Mod}_{q}(\Gamma)\right)^{1 / q}\left(\iint_{0}^{1}|\dot{\gamma}|^{p} \mathrm{~d} s \mathrm{~d} \boldsymbol{\pi}(\gamma)\right)^{1 / p},
$$

which shows that any $\operatorname{Mod}_{q}$-negligible set of curves is also $q$-negligible in the sense of Definition 4.6. This immediately gives that any $q$-upper gradient is a $q$-weak upper gradient, so that

$$
|\nabla f|_{w, q} \leq|\nabla f|_{S, q} \quad \mathfrak{m} \text {-a.e. in } X .
$$

Notice that the combination of (4.4), (4.6) and (4.14) gives (4.1).

\section{Some properties of weak gradients}

In order to close the chain of inequalities in (4.1) we need some properties of the weak gradients introduced in the previous section. The following locality lemma follows by the same arguments as in [6], or by adapting to the case $q \neq 2$ the proof of Lemma 4.4 in [2].

Lemma 5.1 (Pointwise minimality of $|\nabla f|_{*, q}$ ). Let $g_{1}$ and $g_{2}$ be two q-relaxed slopes of $f$. Then $\min \left\{g_{1}, g_{2}\right\}$ is a q-relaxed slope as well. In particular, not only the $L^{q}$ norm of $|\nabla f|_{*, q}$ is minimal, but also $|\nabla f|_{*, q} \leq g$ m-a.e. in $X$ for any relaxed slope $g$ of $f$.

The previous pointwise minimality property immediately yields

$$
|\nabla f|_{*, q} \leq|\nabla f| \quad \mathfrak{m} \text {-a.e. in } X
$$

for any Lipschitz function $f: X \rightarrow \mathbb{R}$.

Also the proof of locality and chain rule is quite standard, see [6] and Proposition 4.8 in [2] for the case $q=2$ (the same proof works in the general case).

Proposition 5.2 (Locality and chain rule). If $f \in L^{q}(X, \mathfrak{m})$ has a $q$-relaxed slope, the following properties hold:

(a) $|\nabla h|_{*, q}=|\nabla f|_{*, q} \mathfrak{m}$-a.e. in $\{h=f\}$ whenever $f$ has a q-relaxed slope.

(b) $|\nabla \phi(f)|_{*, q} \leq\left|\phi^{\prime}(f)\right||\nabla f|_{*, q}$ for any $C^{1}$ and Lipschitz function $\phi$ on an interval containing the image of $f$. Equality holds if $\phi$ is nondecreasing.

Next we consider the stability of $q$-weak upper gradients (as we said, similar properties hold for $q$-upper gradients, see Lemma 4.11 in [20], but we shall not need them here).

Theorem 5.3 (Stability w.r.t. $\mathfrak{m}$-a.e. convergence). Assume that $f_{n}$ are $\mathfrak{m}$-measurable, Sobolev along q-a.e. curve and that $g_{n} \in L^{q}(X, \mathfrak{m})$ are $q$-weak upper gradients of $f_{n}$. Assume furthermore that $f_{n}(x) \rightarrow f(x) \in \mathbb{R}$ for $\mathfrak{m}$-a.e. $x \in X$ and that $\left(g_{n}\right)$ weakly converges to $g$ in $L^{q}(X, \mathfrak{m})$. Then $g$ is a q-weak upper gradient of $f$. 
Proof. Fix a $p$-test plan $\pi$ and let $\theta \in L^{1}(X, \mathfrak{m})$ be strictly positive (its existence is ensured by the $\sigma$-finiteness assumption on $\mathfrak{m})$. By Mazur's theorem we can find convex combinations

$$
h_{n}:=\sum_{i=N_{h}+1}^{N_{h+1}} \alpha_{i} g_{i} \quad \text { with } \alpha_{i} \geq 0, \sum_{i=N_{h}+1}^{N_{h+1}} \alpha_{i}=1, N_{h} \rightarrow \infty
$$

converging strongly to $g$ in $L^{q}(X, \mathfrak{m})$. Denoting by $\tilde{f}_{n}$ the corresponding convex combinations of $f_{n}$, the $h_{n}$ are weak upper gradients of $\tilde{f}_{n}$ and still $\tilde{f}_{n} \rightarrow f \mathfrak{m}$-a.e. in $X$.

Since for every nonnegative Borel function $\varphi: X \rightarrow[0, \infty]$ there holds (with $C=C(\boldsymbol{\pi}))$

$$
\begin{aligned}
\int\left(\int_{\gamma} \varphi\right) \mathrm{d} \boldsymbol{\pi} & =\int\left(\int_{0}^{1} \varphi\left(\gamma_{t}\right)\left|\dot{\gamma}_{t}\right| \mathrm{d} t\right) \mathrm{d} \boldsymbol{\pi} \leq \int\left(\int_{0}^{1} \varphi^{q}\left(\gamma_{t}\right) \mathrm{d} t\right)^{1 / q}\left(\int_{0}^{1}\left|\dot{\gamma}_{t}\right|^{p} \mathrm{~d} t\right)^{1 / p} \mathrm{~d} \boldsymbol{\pi} \\
& \leq\left(\int_{0}^{1} \int \varphi^{q} \mathrm{~d}\left(\mathrm{e}_{t}\right)_{\sharp} \boldsymbol{\pi} \mathrm{d} t\right)^{1 / q}\left(\iint_{0}^{1}\left|\dot{\gamma}_{t}\right|^{p} \mathrm{~d} t \mathrm{~d} \boldsymbol{\pi}\right)^{1 / p} \\
& \leq\left(C \int \varphi^{q} \mathrm{~d} \mathfrak{m}\right)^{1 / q}\left(\iint_{0}^{1}\left|\dot{\gamma}_{t}\right|^{p} \mathrm{~d} t \mathrm{~d} \boldsymbol{\pi}\right)^{1 / p},
\end{aligned}
$$

we obtain, for $\bar{C}:=C^{1 / q}\left(\iint_{0}^{1}\left|\dot{\gamma}_{t}\right|^{p} \mathrm{~d} t \mathrm{~d} \boldsymbol{\pi}\right)^{1 / p}$,

$$
\int\left(\int_{\gamma}\left|h_{n}-g\right|+\min \left\{\left|\tilde{f}_{n}-f\right|, \theta\right\}\right) \mathrm{d} \boldsymbol{\pi} \leq \bar{C}\left(\left\|h_{n}-g\right\|_{q}+\left\|\min \left\{\left|\tilde{f}_{n}-f\right|, \theta\right\}\right\|_{q}\right) \rightarrow 0 .
$$

By a diagonal argument we can find a subsequence $n(k)$ such that

$$
\int_{\gamma}\left|h_{n(k)}-g\right|+\min \left\{\left|\tilde{f}_{n(k)}-f\right|, \theta\right\} \rightarrow 0
$$

as $k \rightarrow \infty$ for $\pi$-a.e. $\gamma$. Since the $\tilde{f}_{n}$ converge $\mathfrak{m}$-a.e. to $f$ and the marginals of $\boldsymbol{\pi}$ are absolutely continuous w.r.t. $\mathfrak{m}$, we have also that for $\boldsymbol{\pi}$-a.e. $\gamma$ there holds $\tilde{f}_{n}\left(\gamma_{0}\right) \rightarrow f\left(\gamma_{0}\right)$ and $\tilde{f}_{n}\left(\gamma_{1}\right) \rightarrow f\left(\gamma_{1}\right)$.

If we fix a curve $\gamma$ satisfying these convergence properties, since $\left(\tilde{f}_{n(k)}\right)_{\gamma}$ are equi-absolutely continuous (their derivatives are bounded by $\left.h_{n(k)} \circ \gamma|\dot{\gamma}|\right)$, and a further subsequence of $\tilde{f}_{n(k)}$ converges a.e. in $[0,1]$ and in $\{0,1\}$ to $f\left(\gamma_{s}\right)$, we can pass to the limit to obtain an absolutely continuous function $f_{\gamma}$ equal to $f\left(\gamma_{s}\right)$ a.e. in $[0,1]$ and in $\{0,1\}$ with derivative bounded by $g\left(\gamma_{s}\right)\left|\dot{\gamma}_{s}\right|$. Since $\boldsymbol{\pi}$ is arbitrary we conclude that $f$ is Sobolev along $q$-a.e. curve and that $h$ is a weak upper gradient of $f$.

It is natural to ask whether $r$-upper gradients really depend on $r$ or not. A natural conjecture is the following: let $r \in(1, \infty)$ and $f: X \rightarrow \mathbb{R}$ Borel. Assume that $\mathfrak{m}$ is a finite measure and that $f$ has a $r$-upper gradient in $L^{r}(X, \mathfrak{m})$. Then, for all $q \in(1, r], f$ has a $q$-upper gradient and $|\nabla f|_{S, q}=|\nabla f|_{S, r} \mathfrak{m}$-a.e. in $X$. 
Notice however that the "converse" implication, namely

$f$ has a $q$-upper gradient in $L^{r}(X, \mathfrak{m}) \Rightarrow f$ has an $r$-upper gradient in $L^{r}(X, \mathfrak{m})$

for $1<q<r<\infty$ does not hold in general. A counterexample has been shown to us by P. Koskela: consider the set $X$ equal to the union of the first and third quadrant in $\mathbb{R}^{2}$, and take as $f$ the characteristic function of the first quadrant. Since the collection of all curves passing from the first to the third quadrant is $\operatorname{Mod}_{2}$-negligible (just take, for $\alpha \in(0,1)$, the family of curves $\rho_{\alpha}(x)=\alpha|x|^{\alpha-1}$, and let $\alpha \downarrow 0)$ it follows that $f$ has a 2 -upper gradient equal to 0 . On the other hand, $f$ is discontinuous along the pencil of curves $\gamma_{\theta}(t):=(2 t-1)(\cos \theta, \sin \theta)$ indexed by $\theta \in[0, \pi / 2]$, and since this family of curves is not $\operatorname{Mod}_{r}$-negligible for $r>2$ it follows that (5.3) fails for $f$. In order to show that the family of curves is not $\operatorname{Mod}_{r}$-negligible for $r>2$, suffices to notice that $\int_{\gamma_{\theta}} g \geq 1$ implies

$$
\frac{1}{2} \leq\left(\int_{0}^{1} g^{r}\left(\gamma_{\theta}(t)\right)|2 t-1| \mathrm{d} t\right)^{1 / r}\left(\int_{0}^{1}|2 t-1|^{-r^{\prime} / r} \mathrm{~d} t\right)^{1 / r^{\prime}} .
$$

Since $r>2$ implies $r^{\prime} / r<1$, integrating both sides over [0, $\left.\pi / 2\right]$ gives a lower bound on the $L^{r}$ norm of $g$ with a positive constant $c(r)$.

In the presence of doubling and a $(1, q)$-Poincaré inequality, (5.3) holds, following the Lipschitz approximation argument in Theorems 4.14 and 4.24 of [6] (we shall not need this fact in the sequel).

\section{Cheeger's functional and its gradient flow}

In this section we assume that $(X, \mathrm{~d})$ is complete and separable and that $\mathfrak{m}$ is a finite Borel measure. As in the previous sections, $q \in(1, \infty)$ and $p$ is the dual exponent. In order to apply the theory of gradient flows of convex functionals in Hilbert spaces, when $q>2$, we need to extend $|\nabla f|_{*, q}$ to functions in $L^{2}(X, \mathfrak{m})$ (because Definition 4.2 was given for $L^{q}(X, \mathfrak{m})$ functions). To this end, we let $f_{N}:=\max \{-N, \min \{f, N\}\}$ and set

$$
\mathcal{C}:=\left\{f: X \rightarrow \mathbb{R}: f_{N} \text { has a } q \text {-relaxed slope for all } N \in \mathbb{N}\right\} .
$$

Accordingly, for all $f \in \mathcal{C}$ we set

$$
|\nabla f|_{*, q}:=\left|\nabla f_{N}\right|_{*, q} \quad \mathfrak{m} \text {-a.e. in }\{|f|<N\}
$$

for all $N \in \mathbb{N}$. We can use the locality property in Proposition 5.2(a) to show that this definition is well posed, up to $\mathfrak{m}$-negligible sets, and consistent with the previous one. Furthermore, locality and chain rules still apply, so we shall not use a different notation for the new gradient.

Although we work with a definition of weak gradient stronger than that of $|\nabla f|_{C, q}$, we call Cheeger's $q$-functional the energy on $L^{2}(X, \mathfrak{m})$ defined by

$$
\mathrm{Ch}_{q}(f):=\frac{1}{q} \int_{X}|\nabla f|_{*, q}^{q} \mathrm{dm},
$$

set to $+\infty$ if $f \in L^{2}(X, \mathfrak{m}) \backslash \mathcal{C}$. 
Theorem 6.1. Cheeger's q-functional $\mathrm{Ch}_{q}$ is convex and lower semicontinuous in $L^{2}(X, \mathfrak{m})$.

Proof. The proof of convexity is elementary, so we focus on lower semicontinuity. Let $\left(f_{n}\right)$ converge to $f$ in $L^{2}(X, \mathfrak{m})$ and assume, after possibly extracting a subsequence and with no loss of generality, that $\mathrm{Ch}_{q}\left(f_{n}\right)$ converges to a finite limit.

Assume first that all the $f_{n}$ have $q$-relaxed slopes, so that $\left|\nabla f_{n}\right|_{*, q}$ is uniformly bounded in $L^{q}(X, \mathfrak{m})$. Let $f_{n(k)}$ be a subsequence such that $\left|\nabla f_{n(k)}\right|_{*, q}$ converges weakly to $g$ in $L^{q}(X, \mathfrak{m})$. Then $g$ is a $q$-relaxed slope of $f$ and

$$
\mathrm{Ch}_{q}(f) \leq \frac{1}{q} \int_{X}|g|^{q} \mathrm{dm} \leq \liminf _{k \rightarrow \infty} \frac{1}{q} \int_{X}\left|\nabla f_{n(k)}\right|_{*, q}^{q} \mathrm{dm}=\liminf _{n \rightarrow \infty} \operatorname{Ch}_{q}\left(f_{n}\right) .
$$

In the general case when $f_{n} \in \mathcal{C}$, we consider the functions

$$
f_{n}^{N}:=\max \left\{-N, \min \left\{f_{n}, N\right\}\right\}
$$

to conclude from the inequality $\left|\nabla f_{n}^{N}\right|_{*, q} \leq\left|\nabla f_{n}\right|_{*, q}$ that

$$
f^{N}:=\max \{-N, \min \{f, N\}\}
$$

has $q$-relaxed slope for any $N \in \mathbb{N}$ and

$$
\int_{X}\left|\nabla f^{N}\right|_{*, q}^{q} \mathrm{~d} \mathfrak{m} \leq \liminf _{n \rightarrow \infty} \int_{X}\left|\nabla f_{n}^{N}\right|_{*, q}^{q} \mathrm{~d} \mathfrak{m} \leq \liminf _{n \rightarrow \infty} \int_{X}\left|\nabla f_{n}\right|_{*, q}^{q} \mathrm{~d} \mathfrak{m} .
$$

Passing to the limit as $N \rightarrow \infty$, the conclusion follows by monotone convergence.

Remark 6.2. More generally, the same argument proves the $L^{2}(X, \mathfrak{m})$-lower semicontinuity of the functional

$$
f \mapsto \int_{X} \frac{|\nabla f|_{*, q}^{q}}{|f|^{\alpha}} \mathrm{dm}
$$

in $\mathcal{C}$, for any $\alpha>0$. Indeed, locality and the chain rule allow the reduction to nonnegative functions $f_{n}$ and we can use the truncation argument of Theorem 6.1 to reduce ourselves to functions with values in an interval $[c, C]$ with $0<c \leq C<\infty$. In this class, we can again use the chain rule to prove the identity

$$
\int_{X}\left|\nabla f^{\beta}\right|_{*, q}^{q} \mathrm{~d} \mathfrak{m}=|\beta|^{q} \int_{X} \frac{|\nabla f|_{*, q}^{q}}{|f|^{\alpha}} \mathrm{d} \mathfrak{m}
$$

with $\beta:=1-\alpha / q$ to obtain the result when $\alpha \neq q$. If $\alpha=q$ we use a logarithmic transformation.

Since the finiteness domain of $\mathrm{Ch}_{q}$ is dense in $L^{2}(X, \mathfrak{m})$ (it includes bounded Lipschitz functions), the Hilbertian theory of gradient flows (see for instance [5] or [1]) can be applied to Cheeger's functional (6.3) to provide, for all $f_{0} \in L^{2}(X, \mathfrak{m})$, a locally absolutely continuous map $t \mapsto f_{t}$ from $(0, \infty)$ to $L^{2}(X, \mathfrak{m})$, with $f_{t} \rightarrow f_{0}$ as $t \downarrow 0$, whose derivative satisfies

$$
\frac{d}{d t} f_{t} \in-\partial^{-} \mathrm{Ch}_{q}\left(f_{t}\right) \quad \text { for a.e. } t \in(0, \infty) \text {. }
$$


Having in mind the regularizing effect of gradient flows, namely the selection of elements with minimal $L^{2}(X, \mathfrak{m})$ norm in $\partial^{-} \mathrm{Ch}_{q}$, the following definition is natural.

Definition 6.3 ( $q$-Laplacian). The $q$-Laplacian $\Delta_{q} f$ of $f \in L^{2}(X, \mathfrak{m})$ is defined for those $f$ such that $\partial^{-} \mathrm{Ch}_{q}(f) \neq \emptyset$. For those $f,-\Delta_{q} f$ is the element of minimal $L^{2}(X, \mathfrak{m})$ norm in $\partial^{-} \mathrm{Ch}_{q}(f)$. The domain of $\Delta_{q}$ will be denoted by $D\left(\Delta_{q}\right)$.

Remark 6.4 (Potential lack of linearity). It should be observed that, even in the case $q=2$, in general the Laplacian is not a linear operator. Still, the trivial implication

$$
v \in \partial^{-} \mathrm{Ch}_{q}(f) \quad \Longrightarrow \quad \lambda^{q-1} v \in \partial^{-} \mathrm{Ch}_{q}(\lambda f), \quad \forall \lambda \in \mathbb{R},
$$

ensures that the $q$-Laplacian (and so the gradient flow of $\mathrm{Ch}_{q}$ ) is $(q-1)$-homogenous.

We can now write

$$
\frac{\mathrm{d}}{\mathrm{d} t} f_{t}=\Delta_{q} f_{t}
$$

for gradient flows $f_{t}$ of $\mathrm{Ch}_{q}$, the derivative being understood to be in $L^{2}(X, \mathfrak{m})$, in accordance with the classical case.

Proposition 6.5 (Integration by parts). For all $f \in D\left(\Delta_{q}\right)$ and $g \in D\left(\mathrm{Ch}_{q}\right)$ there holds

$$
-\int_{X} g \Delta_{q} f \mathrm{~d} \mathfrak{m} \leq \int_{X}|\nabla g|_{*, q}|\nabla f|_{*, q}^{q-1} \mathrm{~d} \mathfrak{m}
$$

Equality holds if $g=\phi(f)$ with $\phi \in C^{1}(\mathbb{R})$ with bounded derivative on the image of $f$.

Proof. Since $-\Delta_{q} f \in \partial^{-} \mathrm{Ch}_{q}(f)$ there holds

$$
\mathrm{Ch}_{q}(f)-\int_{X} \varepsilon g \Delta_{q} f \mathrm{~d} \mathfrak{m} \leq \mathrm{Ch}_{q}(f+\varepsilon g), \quad \forall g \in L^{q}(X, \mathfrak{m}), \varepsilon \in \mathbb{R} .
$$

For $\varepsilon>0,|\nabla f|_{*, q}+\varepsilon|\nabla g|_{*, q}$ is a $q$-relaxed slope of $f+\varepsilon g$ (possibly not minimal) whenever $f$ and $g$ have $q$-relaxed slopes. By truncation, it is immediate to obtain from this fact that $f, g \in \mathcal{C}$ implies $f+\varepsilon g \in \mathcal{C}$ and

$$
|\nabla(f+\varepsilon g)|_{*, q} \leq|\nabla f|_{*, q}+\varepsilon|\nabla g|_{*, q} \quad \mathfrak{m} \text {-a.e. in } X .
$$

Thus there holds $q \mathrm{Ch}_{q}(f+\varepsilon g) \leq \int_{X}\left(|\nabla f|_{*, q}+\varepsilon|\nabla g|_{*, q}\right)^{q}$ dm , and therefore

$$
\begin{aligned}
-\int_{X} \varepsilon g \Delta_{q} f \mathrm{~d} \mathfrak{m} & \leq \frac{1}{q} \int_{X}\left(|\nabla f|_{*, q}+\varepsilon|\nabla g|_{*, q}\right)^{q}-|\nabla f|_{*, q}^{q} \mathrm{~d} \mathfrak{m} \\
& =\varepsilon \int_{X}|\nabla g|_{*, q}|\nabla f|_{*, q}^{q-1} \mathrm{~d} \mathfrak{m}+o(\varepsilon) .
\end{aligned}
$$

Dividing by $\varepsilon$ and letting $\varepsilon \downarrow 0$ we get (6.5). 
For the second statement we recall that $|\nabla(f+\varepsilon \phi(f))|_{*, q}=\left(1+\varepsilon \phi^{\prime}(f)\right)|\nabla f|_{*, q}$ for $|\varepsilon|$ small enough. Hence

$$
\begin{aligned}
\mathrm{Ch}_{q}(f+\varepsilon \phi(f))-\mathrm{Ch}_{q}(f) & =\frac{1}{q} \int_{X}|\nabla f|_{*, q}^{q}\left(\left(1+\varepsilon \phi^{\prime}(f)\right)^{q}-1\right) \mathrm{d} \mathfrak{m} \\
& =\varepsilon \int_{X}|\nabla f|_{*, q}^{q} \phi^{\prime}(f) \mathrm{d} \mathfrak{m}+o(\varepsilon),
\end{aligned}
$$

which implies that $\int_{X} v \phi(f) \mathrm{d} \mathfrak{m}=\int_{X}|\nabla f|_{*, q}^{q} \phi^{\prime}(f) \mathrm{dm}$ for any $v \in \partial^{-} \operatorname{Ch}_{q}(f)$, and gives the claim with $v=-\Delta_{q} f$.

Proposition 6.6 (Some properties of the gradient flow of $\left.\mathrm{Ch}_{q}\right)$. Let $f_{0} \in L^{2}(X, \mathfrak{m})$ and let $\left(f_{t}\right)$ be the gradient flow of $\mathrm{Ch}_{q}$ starting from $f_{0}$. Then the following properties hold:

- (Mass preservation) $\int f_{t} \mathrm{~d} \mathfrak{m}=\int f_{0} \mathrm{~d} \mathfrak{m}$ for any $t \geq 0$.

- (Maximum principle) If $f_{0} \leq C$ (respectively, $f_{0} \geq c$ ) $\mathfrak{m}$-a.e. in $X$, then $f_{t} \leq C$ (respectively, $\left.f_{t} \geq c\right) \mathfrak{m}$-a.e. in $X$ for any $t \geq 0$.

- (Energy dissipation) Suppose $0<c \leq f_{0} \leq C<\infty \mathfrak{m}$-a.e. in $X$ and $\Phi \in$ $C^{2}([c, C])$. Then $t \mapsto \int \Phi\left(f_{t}\right) \mathrm{dm}$ is locally absolutely continuous in $(0, \infty)$ and there holds

$$
\frac{\mathrm{d}}{\mathrm{d} t} \int \Phi\left(f_{t}\right) \mathrm{d} \mathfrak{m}=-\int \Phi^{\prime \prime}\left(f_{t}\right)\left|\nabla f_{t}\right|_{*, q}^{q} \mathrm{~d} \mathfrak{m} \quad \text { for a.e. } t \in(0, \infty) .
$$

Proof. (Mass preservation) Just observe that from (6.5) we get

$$
\left|\frac{\mathrm{d}}{\mathrm{d} t} \int f_{t} \mathrm{~d} \mathfrak{m}\right|=\left|\int \mathbf{1} \cdot \Delta_{q} f_{t} \mathrm{~d} \mathfrak{m}\right| \leq \int|\nabla \mathbf{1}|_{*, q}\left|\nabla f_{t}\right|_{*, q}^{q} \mathrm{~d} \mathfrak{m}=0 \quad \text { for a.e. } t>0,
$$

where $\mathbf{1}$ is the function identically equal to 1 , which has minimal $q$-relaxed slope equal to 0 by (5.1).

(Maximum principle) Fix $f \in L^{2}(X, \mathfrak{m})$ and $\tau>0$. Following the so-called implicit Euler scheme, let $f^{\tau}$ be the unique minimizer of

$$
g \mapsto \mathrm{Ch}_{q}(g)+\frac{1}{2 \tau} \int_{X}|g-f|^{2} \mathrm{dm} .
$$

Assume that $f \leq C$. We claim that in this case $f^{\tau} \leq C$ as well. Indeed, if this is not the case we can consider the competitor $g:=\min \left\{f^{\tau}, C\right\}$ in the above minimization problem. By locality we get $\mathrm{Ch}(g) \leq \mathrm{Ch}\left(f^{\tau}\right)$ and the $L^{2}$ distance between $f$ and $g$ is strictly smaller than that between $f$ and $f^{\tau}$ as soon as $\mathfrak{m}\left(\left\{f^{\tau}>C\right\}\right)>0$, which is a contradiction. Starting from $f_{0}$, iterating this procedure, and using the fact that the implicit Euler scheme converges as $\tau \downarrow 0$ to the gradient flow (see [5] and [1] for details), we get the conclusion.

(Energy dissipation) Since $t \mapsto f_{t} \in L^{2}(X, \mathfrak{m})$ is locally absolutely continuous and, by the maximum principle, the $f_{t}$ take their values in $[c, C] \mathfrak{m}$-a.e., from the fact that $\Phi$ is Lipschitz in $[c, C]$ we get the claimed absolute continuity. Now notice that we have $\frac{\mathrm{d}}{\mathrm{d} t} \int \Phi\left(f_{t}\right) \mathrm{d} \mathfrak{m}=\int \Phi^{\prime}\left(f_{t}\right) \Delta_{q} f_{t} \mathrm{~d} \mathfrak{m}$ for a.e. $t>0$. Since $\Phi^{\prime}$ belongs to $C^{1}([c, C])$, from (6.5) with $g=\Phi^{\prime}\left(f_{t}\right)$ we get the conclusion. 


\section{Equivalence of gradients}

In this section we prove the equivalence of weak gradients. We assume that $(X, \mathrm{~d})$ is compact (this assumption is used to be able to apply the results of Section 3 and in Lemma 7.2, to apply (2.2)) and that $\mathfrak{m}$ is a finite Borel measure, so that the $L^{2}$-gradient flow of $\mathrm{Ch}_{q}$ can be used.

We start with the following proposition, which relates energy dissipation to a (sharp) combination of $q$-weak gradients and metric dissipation in $W_{p}$.

Proposition 7.1. Let $\mu_{t}=f_{t} \mathfrak{m}$ be a curve in $A C^{p}\left([0,1],\left(\mathscr{P}(X), W_{p}\right)\right)$. Assume that for some $0<c<C<\infty$ there holds $c \leq f_{t} \leq C \mathfrak{m}$-a.e. in $X$ for any $t \in[0,1]$, and that $f_{0}$ is Sobolev along q-a.e. curve with $\left|\nabla f_{0}\right|_{w, q} \in L^{q}(X, \mathfrak{m})$. Then for all $\Phi \in C^{2}([c, C])$ convex there holds, $\forall t>0$,

$$
\int \Phi\left(f_{0}\right) \mathrm{d} \mathfrak{m}-\int \Phi\left(f_{t}\right) \mathrm{d} \mathfrak{m} \leq \frac{1}{q} \iint_{0}^{t}\left(\Phi^{\prime \prime}\left(f_{0}\right)\left|\nabla f_{0}\right| w, q\right)^{q} f_{s} \mathrm{~d} s \mathrm{~d} \mathfrak{m}+\frac{1}{p} \int_{0}^{t}\left|\dot{\mu}_{s}\right|^{p} \mathrm{~d} s .
$$

Proof. Let $\pi \in \mathscr{P}(C([0,1], X))$ be a plan associated to the curve $\left(\mu_{t}\right)$ as in Proposition 2.2. The assumption $f_{t} \leq C$ m-a.e. and the fact that $\iint_{0}^{1}\left|\dot{\gamma}_{t}\right|^{p} \mathrm{~d} t \mathrm{~d} \boldsymbol{\pi}(\gamma)=$ $\int\left|\dot{\mu}_{t}\right|^{p} \mathrm{~d} t<\infty$ guarantee that $\pi$ is a $p$-test plan. Now notice that there holds $\left|\nabla \Phi^{\prime}\left(f_{0}\right)\right|_{w, q}=\Phi^{\prime \prime}\left(f_{0}\right)\left|\nabla f_{0}\right|_{w, q}$ (this follows easily from the characterization (4.11)). Thus we get

$$
\begin{aligned}
\int \Phi\left(f_{0}\right) & -\int \Phi\left(f_{t}\right) \mathrm{d} \mathfrak{m} \leq \int \Phi^{\prime}\left(f_{0}\right)\left(f_{0}-f_{t}\right) \mathrm{d} \mathfrak{m}=\int \Phi^{\prime}\left(f_{0}\right) \circ \mathrm{e}_{0}-\Phi^{\prime}\left(f_{0}\right) \circ \mathrm{e}_{t} \mathrm{~d} \boldsymbol{\pi} \\
& \leq \iint_{0}^{t} \Phi^{\prime \prime}\left(f_{0}\left(\gamma_{s}\right)\right)\left|\nabla f_{0}\right|_{w, q}\left(\gamma_{s}\right)\left|\dot{\gamma}_{s}\right| \mathrm{d} s \mathrm{~d} \boldsymbol{\pi}(\gamma) \\
& \leq \frac{1}{q} \iint_{0}^{t}\left(\Phi^{\prime \prime}\left(f_{0}\left(\gamma_{s}\right)\right)\left|\nabla f_{0}\right|_{w, q}\left(\gamma_{s}\right)\right)^{q} \mathrm{~d} s \mathrm{~d} \boldsymbol{\pi}(\gamma)+\frac{1}{p} \iint_{0}^{t}\left|\dot{\gamma}_{s}\right|^{p} \mathrm{~d} s \mathrm{~d} \boldsymbol{\pi}(\gamma) \\
& =\frac{1}{q} \iint_{0}^{t}\left(\Phi^{\prime \prime}\left(f_{0}\right)\left|\nabla f_{0}\right|_{w, q}\right)^{q} f_{s} \mathrm{~d} s \mathrm{~d} \mathfrak{m}+\frac{1}{p} \int_{0}^{t}\left|\dot{\mu}_{s}\right|^{p} \mathrm{~d} s .
\end{aligned}
$$

The key argument for achieving the identification is the following lemma, which gives a sharp bound on the $W_{p}$-speed of the $L^{2}$-gradient flow of $\mathrm{Ch}_{q}$. This lemma was introduced in [17] and then used in [9] and [2] to study the heat flow on metric measure spaces.

Lemma 7.2 (Kuwada's lemma). Let $f_{0} \in L^{q}(X, \mathfrak{m})$ and let $\left(f_{t}\right)$ be the gradient flow of $\mathrm{Ch}_{q}$ starting from $f_{0}$. Assume that for some $0<c<C<\infty$ there holds $c \leq f_{0} \leq C \mathfrak{m}$-a.e. in $X$, and that $\int f_{0} \mathrm{~d} \mathfrak{m}=1$. Then the curve $t \mapsto \mu_{t}:=f_{t} \mathfrak{m} \in$ $\mathscr{P}(X)$ is absolutely continuous w.r.t. $W_{p}$ and there holds

$$
\left|\dot{\mu}_{t}\right|^{p} \leq \int \frac{\left|\nabla f_{t}\right|_{*, q}^{q}}{f_{t}^{p-1}} \mathrm{dm} \quad \text { for a.e. } t \in(0, \infty)
$$


Proof. We start from the duality formula (2.2) (written with $\varphi=-\psi$ ):

$$
\frac{W_{p}^{p}(\mu, \nu)}{p}=\sup _{\varphi \in \operatorname{Lip}(X)} \int_{X} Q_{1} \varphi d \nu-\int_{X} \varphi d \mu
$$

where $Q_{t} \varphi$ is defined in (3.1) and (3.2), so that $Q_{1} \varphi=\psi^{c}$. Fix $\varphi \in \operatorname{Lip}(X)$ and recall (Proposition 3.3) that the map $t \mapsto Q_{t} \varphi$ is Lipschitz with values in $C(X)$, in particular also as a $L^{2}(X, \mathfrak{m})$-valued map.

Fix also $0 \leq t<s$, set $\ell=(s-t)$, and recall that since $\left(f_{t}\right)$ is a gradient flow of $\mathrm{Ch}_{q}$ in $L^{2}(X, \mathfrak{m})$, the map $[0, \ell] \ni \tau \mapsto f_{t+\tau}$ is absolutely continuous with values in $L^{2}(X, \mathfrak{m})$. Therefore, since both factors are uniformly bounded, the map $[0, \ell] \ni \tau \mapsto Q_{\frac{\tau}{\ell}} \varphi f_{t+\tau}$ is absolutely continuous with values in $L^{2}(X, \mathfrak{m})$. In addition, the equality

$$
\frac{Q_{\frac{\tau+h}{\ell}} \varphi f_{t+\tau+h}-Q_{\frac{\tau}{\ell}} \varphi f_{t+\tau}}{h}=f_{t+\tau} \frac{Q_{\frac{\tau+h}{\ell}}-Q_{\frac{\tau}{\ell}} \varphi}{h}+Q_{\frac{\tau+h}{\ell}} \varphi \frac{f_{t+\tau+h}-f_{t+\tau}}{h}
$$

together with the uniform continuity of $(x, \tau) \mapsto Q_{\frac{\tau}{\ell}} \varphi(x)$ shows that the derivative of $\tau \mapsto Q_{\frac{\tau}{\ell}} \varphi f_{t+\tau}$ can be computed via the Leibniz rule.

We have:

$$
\begin{aligned}
\int_{X} Q_{1} \varphi \mathrm{d} \mu_{s}-\int_{X} \varphi \mathrm{d} \mu_{t} & =\int Q_{1} \varphi f_{t+\ell} \mathrm{d} \mathfrak{m}-\int_{X} \varphi f_{t} \mathrm{~d} \mathfrak{m} \\
& =\int_{X} \int_{0}^{\ell} \frac{\mathrm{d}}{\mathrm{d} \tau}\left(Q_{\frac{\tau}{\ell}} \varphi f_{t+\tau}\right) \mathrm{d} \tau \mathrm{d} \mathfrak{m} \\
& \leq \int_{X} \int_{0}^{\ell}-\frac{\left|\nabla Q_{\frac{\tau}{\ell}} \varphi\right|^{q}}{q \ell} f_{t+\tau}+Q_{\frac{\tau}{\ell}} \varphi \Delta_{q} f_{t+\tau} \mathrm{d} \tau \mathrm{d} \mathfrak{m},
\end{aligned}
$$

in which we have used Theorem 3.5.

Observe that by inequalities (6.5) and (5.1) we have

$$
\begin{aligned}
\int_{X} Q_{\frac{\tau}{\ell}} \varphi \Delta_{q} f_{t+\tau} \mathrm{d} \mathfrak{m} & \leq \int_{X}\left|\nabla Q_{\frac{\tau}{\ell}} \varphi\right|_{*, q}\left|\nabla f_{t+\tau}\right|_{*, q}^{q-1} \mathrm{~d} \mathfrak{m} \leq \int_{X}\left|\nabla Q_{\frac{\tau}{\ell}} \varphi\right|\left|\nabla f_{t+\tau}\right|_{*, q}^{q-1} \mathrm{~d} \mathfrak{m} \\
& \leq \frac{1}{q \ell} \int_{X}\left|\nabla Q_{\frac{\tau}{\ell}} \varphi\right|^{q} f_{t+\tau} d \mathfrak{m}+\frac{\ell^{p-1}}{p} \int_{X} \frac{\left|\nabla f_{t+\tau}\right|_{*, q}^{q}}{f_{t+\tau}^{p-1}} \mathrm{dm} .
\end{aligned}
$$

Plugging this inequality into (7.2), we obtain

$$
\int_{X} Q_{1} \varphi \mathrm{d} \mu_{s}-\int_{X} \varphi \mathrm{d} \mu_{t} \leq \frac{\ell^{p-1}}{p} \int_{0}^{\ell} \int_{X} \frac{\left|\nabla f_{t+\tau}\right|_{*, q}^{q}}{f_{t+\tau}^{p-1}} \mathrm{dm} .
$$

This latter bound does not depend on $\varphi$, so from (7.1) we deduce

$$
W_{p}^{p}\left(\mu_{t}, \mu_{s}\right) \leq \ell^{p-1} \int_{0}^{\ell} \int_{X} \frac{\left|\nabla f_{t+\tau}\right|_{*, q}^{q}}{f_{t+\tau}^{p-1}} \mathrm{dm}
$$

At Lebesgue points of $r \mapsto \int_{X}\left|\nabla f_{r}\right|_{*, q}^{q} / f_{r}^{p-1} \mathrm{dm}$ where the metric speed exists we obtain the stated pointwise bound on the metric speed. 
The following result provides equivalence between weak and relaxed gradients. Recall the set $\mathcal{C}$ defined in (6.1).

Theorem 7.3. Let $f: X \rightarrow \mathbb{R}$ be Borel. Assume that $f$ is Sobolev along q-a.e. curve and that $|\nabla f|_{w, q} \in L^{q}(X, \mathfrak{m})$. Then $f \in \mathcal{C}$ and $|\nabla f|_{*, q}=|\nabla f|_{w, q} \mathfrak{m}$-a.e. in $X$.

Proof. Up to a truncation argument and the addition of a constant, we can assume that $0<c \leq f \leq C<\infty$ m-a.e. for some $0<c \leq C<\infty$. Let $\left(g_{t}\right)$ be the $L^{2}$ gradient flow of $\mathrm{Ch}_{q}$ starting from $g_{0}:=f$ and let us choose $\Phi \in C^{2}([c, C])$ in such a way that $\Phi^{\prime \prime}(z)=z^{1-p}$ in $[c, C]$. Recall that $c \leq g_{t} \leq C \mathfrak{m}$-a.e. in $X$ and that from Proposition 6.6 we have

$$
\int \Phi\left(g_{0}\right) \mathrm{d} \mathfrak{m}-\int \Phi\left(g_{t}\right) \mathrm{d} \mathfrak{m}=\int_{0}^{t} \int_{X} \Phi^{\prime \prime}\left(g_{s}\right)\left|\nabla g_{s}\right|_{*, q}^{q} \mathrm{~d} \mathfrak{m} \mathrm{d} s \quad \forall t \in[0, \infty) .
$$

In particular this gives that $\int_{0}^{\infty} \int_{X} \Phi^{\prime \prime}\left(g_{s}\right)\left|\nabla g_{s}\right|_{*, q}^{q} \mathrm{dm} \mathrm{d} s$ is finite. Setting $\mu_{t}=g_{t} \mathfrak{m}$, Lemma 7.2 and the lower bound on $g_{t}$ give that $\mu_{t} \in A C^{p}\left((0, \infty),\left(\mathscr{P}(X), W_{p}\right)\right)$, so that Proposition 7.1 and Lemma 7.2 yield

$$
\begin{aligned}
& \int \Phi\left(g_{0}\right) \mathrm{d} \mathfrak{m}-\int \Phi\left(g_{t}\right) \mathrm{d} \mathfrak{m} \leq \frac{1}{q} \int_{0}^{t} \int_{X}\left(\Phi^{\prime \prime}\left(g_{0}\right)\left|\nabla g_{0}\right|_{w, q}\right)^{q} g_{s} \mathrm{~d} \mathfrak{m} \mathrm{d} s \\
& +\frac{1}{p} \int_{0}^{t} \int_{X} \frac{\left|\nabla g_{s}\right|_{*, q}^{q}}{g_{s}^{p-1}} \mathrm{~d} \mathfrak{m} \mathrm{d} s .
\end{aligned}
$$

Hence, comparing this last expression with (7.4), our choice of $\Phi$ gives

$$
\frac{1}{q} \iint_{0}^{t} \frac{\left|\nabla g_{s}\right|_{*, q}^{q}}{g_{s}^{p-1}} \mathrm{~d} s \mathrm{~d} \mathfrak{m} \leq \int_{0}^{t} \int_{X} \frac{1}{q}\left(\frac{\left|\nabla g_{0}\right|_{w, q}}{g_{0}^{p-1}}\right)^{q} g_{s} \mathrm{~d} \mathfrak{m} \mathrm{d} s .
$$

Now, the bound $f \geq c>0$ ensures $\Phi^{\prime \prime}\left(g_{0}\right)\left|\nabla g_{0}\right|_{*, q} \in L^{q}(X, \mathfrak{m})$. In addition, the maximum principle together with the convergence of $g_{s}$ to $g_{0}$ in $L^{2}(X, \mathfrak{m})$ as $s \downarrow 0$ yields that the convergence is also weak* in $L^{\infty}(X, \mathfrak{m})$. Therefore,

$$
\limsup _{t \downarrow 0} \frac{1}{t} \iint_{0}^{t} \frac{\left|\nabla g_{s}\right|_{*, q}^{q}}{g_{s}^{p-1}} \mathrm{~d} s \mathrm{~d} \mathfrak{m} \leq \int_{X} \frac{\left|\nabla g_{0}\right|_{w, q}^{q}}{g_{0}^{q(p-1)}} g_{0} \mathrm{~d} \mathfrak{m}=\int_{X} \frac{\left|\nabla g_{0}\right|_{w, q}^{q}}{g_{0}^{p-1}} \mathrm{~d} \mathfrak{m} .
$$

The lower semicontinuity property stated in Remark 6.2 with $\alpha=p-1$ then gives

$$
\int_{X} \frac{\left|\nabla g_{0}\right|_{*, q}^{q}}{g_{0}^{p-1}} \mathrm{~d} \mathfrak{m} \leq \int_{X} \frac{\left|\nabla g_{0}\right|_{w, q}^{q}}{g_{0}^{p-1}} \mathrm{~d} \mathfrak{m} .
$$

This, together with the inequality $\left|\nabla g_{0}\right|_{w, q} \leq\left|\nabla g_{0}\right|_{*, q}$ m-a.e. in $X$, gives the conclusion.

In particular, taking into account (4.1), we obtain the following equivalence result. We state it for $L^{q}(X, \mathfrak{m})$ functions because in the definition of $q$-relaxed upper gradient and $q$-relaxed slope this integrability assumption is made (see also Remark 4.4), while no integrability is made in the other two definitions. It is also clear that if we extend the "relaxed" definitions of gradient by truncation, as in (6.2), then equivalence goes beyond $L^{q}(X, \mathfrak{m})$ functions. 
Theorem 7.4 (Equivalence of weak gradients and independence of $q$ ). Let $f \in$ $L^{q}(X, \mathfrak{m})$. Then the following four properties are equivalent:

(i) $f$ has a q-relaxed upper gradient;

(ii) $f$ has a q-relaxed slope;

(iii) $f$ has a q-upper gradient in $L^{q}(X, \mathfrak{m})$;

(iv) $f$ has a q-weak upper gradient in $L^{q}(X, \mathfrak{m})$.

In addition, the minimal q-relaxed upper gradient, the minimal q-relaxed slope, and the minimal q-upper and q-weak upper gradients coincide $\mathfrak{m}$-a.e. in $X$.

Proof. If either of the four properties holds for some gradient $g$, then (4.1) gives that $f$ is Sobolev along $q$-a.e. curve and $|\nabla f|_{w, q} \leq g \mathfrak{m}$-a.e. in $X$. Then, Theorem 7.3 yields $|\nabla f|_{*, q} \leq g \mathfrak{m}$-a.e. in $X$ and we can invoke (4.1) again to obtain that all four properties hold and the corresponding weak gradients are equal.

\section{Further comments and extensions}

In this section we point out how our main results, namely Theorems 7.3 and 7.4 , can be extended to more general metric measure spaces. Recall that, in the previous section, we derived them under the assumptions that $(X, \mathrm{~d})$ is a compact metric space and that $\mathfrak{m}$ is a finite measure.

\subsection{The role of the compactness assumption in Section 3}

The compactness assumption is not really needed, and it suffices to assume that $(X, \mathrm{~d})$ is a complete metric space. The only difference appears at the level of the definition of $D^{ \pm}(x, t)$, since in this case existence of minimizers is not guaranteed, and one has to work with minimizing sequences. This results in longer proofs, but the arguments remain essentially the same; see [2] for a detailed proof in the case $p=q=2$. Thanks to this remark, the proof of the equivalence results extends immediately to complete and separable metric measure spaces with $(X, \mathrm{~d}, \mathfrak{m})$ with $\mathrm{d}$ bounded and $\mathfrak{m}$ finite.

Also, it is worthwhile to remark that all the results (except of course the Lipschitz bounds on $Q_{t} f$ and the continuity of $t \mapsto Q_{t} f$ from $[0, \infty)$ to $\left.C(X)\right)$ of Section 3 remain valid for lower semicontinuous functions $f: X \rightarrow \mathbb{R} \cup\{+\infty\}$ satisfying

$$
f(x) \geq-C\left(1+\mathrm{d}^{r}(x, \bar{x})\right) \quad \forall x \in X
$$

for suitable $\bar{x} \in X, C \geq 0$, and $r \in[0, p)$.

\subsection{Locally finite metric measure spaces}

We say that a metric measure space $(X, \mathrm{~d}, \mathfrak{m})$ is locally finite if $(X, \mathrm{~d})$ is complete and separable and any $x \in \operatorname{supp} \mathfrak{m}$ has a neighbourhood $U$ with finite $\mathfrak{m}$-measure.

For any locally finite metric measure space it is not difficult to find (choosing for instance as $U$ balls with $\mathfrak{m}$-negligible boundary) a nondecreasing sequence of 
open sets $A_{h}$ whose union covers m-almost all of $X$ and whose boundaries $\partial A_{h}$ are $\mathfrak{m}$-negligible. Then, setting $X_{h}=\overline{A_{h}}$, we can apply the equivalence results in all metric measure spaces $\left(X_{h}, \mathrm{~d}, \mathfrak{m}\right)$ to obtain the equivalence in $(X, \mathrm{~d}, \mathfrak{m})$. This is due to the fact that the minimal $q$-weak upper gradient satisfies the following local-to-global property (see Theorem 4.20 in [3] for a proof in the case $p=q=2$ ):

$$
|\nabla f|_{X, w, q}=|\nabla f|_{X_{h}, w, q} \quad \mathfrak{m} \text {-a.e. in } X_{h} .
$$

An analogous property holds for the larger gradient, namely the minimal $q$-relaxed slope (arguing as in Lemma 4.11 of [2]):

$$
|\nabla f|_{X, *, q}=|\nabla f|_{X_{h}, *, q} \quad \mathfrak{m} \text {-a.e. in } X_{h}
$$

Combining (8.1) and (8.2) gives the identification result for all gradients and all locally finite metric measure spaces.

\subsection{An enforcement of the density result}

In Theorem 7.3 we proved that if $f: X \rightarrow \mathbb{R}$ is Borel, $f$ is Sobolev along $q$-a.e. curve and $|\nabla f|_{w, q} \in L^{q}(X, \mathfrak{m})$, then there exist Lipschitz functions $f_{n}$ convergent to $f \mathfrak{m}$-a.e. in $X$ and satisfying

$$
\left|\nabla f_{n}\right| \rightarrow|\nabla f|_{w, q} \quad \text { in } L^{q}(X, \mathfrak{m})
$$

This follows by a diagonal argument, thanks to the fact that all truncations $f_{N}$ of $f$ satisfy $\mathrm{Ch}_{q}\left(f_{N}\right) \leq \frac{1}{q} \int_{X}|\nabla f|_{w, q}^{q} \mathrm{dm}$. It is worthwhile to notice that $(8.3)$ can be improved by asking for the existence of Lipschitz functions $f_{n}$ such that $\operatorname{Lip}_{a}\left(f_{n}, \cdot\right) \rightarrow|\nabla f|_{w, q}$ in $L^{q}(X, \mathfrak{m})$, where $\operatorname{Lip}_{a}(f, \cdot)$ is the asymptotic Lipschitz constant defined in (3.7). The key observation is that, as noticed in (3.10), the Hamilton-Jacobi subsolution property holds with the new, and larger, pseudo gradient $\operatorname{Lip}_{a}(g, \cdot)$. Starting from this observation, and using the convexity inequality

$\operatorname{Lip}_{a}((1-\chi) f+\chi g) \leq(1-\chi(x)) \operatorname{Lip}_{a}(f, x)+\chi(x) \operatorname{Lip}_{a}(g, x)+\operatorname{Lip}(\chi)|f(x)-g(x)|$

for Lipschitz $\chi: X \rightarrow[0,1]$ and Lipschitz $f, g: X \rightarrow \mathbb{R}$, one can build Cheeger's energy by minimizing the integrals of $\operatorname{Lip}_{a}\left(f_{n}, \cdot\right)$ instead of the integral of $|\nabla g|$, still getting a convex and lower semicontinuous functional and a corresponding relaxed gradient. Then, (3.10) provides Kuwada's Lemma 7.2 for the new Cheeger energy and the proof of Theorem 7.3 can repeated word by word.

\subsection{Orlicz-Wasserstein spaces}

Another potential extension, that we shall not develop here, is for general Lagrangians-Hamiltonians. One can consider the functions

$$
Q_{t} f(x):=\inf _{y \in X} f(y)+t L\left(\frac{\mathrm{d}(y, x)}{t}\right)
$$


and prove that $\frac{\mathrm{d}}{\mathrm{d} t} Q_{t} f+H\left(\nabla Q_{t} f\right) \leq 0$ with $H=L^{*}$. In this way, gradients in Orlicz spaces such as $L \log L$ could also be considered. On the other hand, the Orlicz-Wasserstein distances

$$
W_{L}(\mu, \nu):=\inf \left\{\lambda>0: \inf _{\boldsymbol{\pi} \in \Gamma(\mu, \nu)} \int L\left(\frac{\mathrm{d}(x, y)}{\lambda}\right) \mathrm{d} \boldsymbol{\pi} \leq 1\right\}
$$

have not been considered much so far (except in [21] and more implicitly in [7] and [22]) and the extension of Lisini's superposition theorem to this class of distances is not known, although expected to be true. Such extensions might be particularly interesting to deal with the limiting case $q \downarrow 1$, where the Wasserstein exponent $p$ goes to $\infty$ (for instance $L \log L$ integrability of gradients corresponds to exponential integrability of the metric derivative on curves).

\section{5. $W^{1,1}$ and $B V$ spaces}

In this subsection we discuss the limiting case $q=1, p=\infty$, and assume for the sake of simplicity that $(X, \mathrm{~d})$ is locally compact and separable. Following the approach in [19], for any open set $A \subset X$ we can define

$$
|D f|(A):=\inf \left\{\liminf _{h \rightarrow \infty} \int_{A}\left|\nabla f_{h}\right| \mathrm{dm}: f_{h} \in \operatorname{Lip}_{\mathrm{loc}}(A), f_{h} \rightarrow f \text { in } L_{\mathrm{loc}}^{1}(A)\right\} .
$$

It is possible to show that, whenever $|D f|(X)<\infty$, the set function $A \mapsto|D f|(A)$ is the restriction to open sets of $X$ of a finite Borel measure, that we still denote by $|D f|$. In the case when $|D f|$ is absolutely continuous with respect to $\mathfrak{m}$, corresponding to the Sobolev space $W^{1,1}$ we may define $|\nabla f|_{*, 1}$ as the density of $|D f|$ with respect to $\mathfrak{m}$.

This approach corresponds to 1-relaxed slopes. Coming to 1-weak upper gradients, it is natural to consider $\infty$-test plans as probability measures $\boldsymbol{\pi}$ concentrated on Lipschitz curves and to define exceptional sets of curves using this class of test plans. Then the class of functions which are $B V$ along 1-almost every curve can be defined. It is not hard to show that if $|D f|(X)<\infty$ and $\pi$ is a $\infty$-test plan such that $\left(\mathrm{e}_{t}\right)_{\#} \boldsymbol{\pi} \leq C(\boldsymbol{\pi}) \mathfrak{m}$ for all $t \in[0,1]$, then there holds the following inequality between measures in $X$ :

$$
\int \gamma_{\sharp}|D(f \circ \gamma)| d \boldsymbol{\pi}(\gamma) \leq C(\boldsymbol{\pi})\|\operatorname{Lip}(\gamma)\|_{L^{\infty}(\boldsymbol{\pi})}|D f|,
$$

where $|D(f \circ \gamma)|$ is the total variation measure of the map $f \circ \gamma:[0,1] \rightarrow \mathbb{R}$. This provides one connection between 1-weak upper gradients and 1-relaxed slopes, while in [4] the arguments of this paper are adapted to show that the supremum of

$$
\frac{1}{C(\boldsymbol{\pi})\|\operatorname{Lip}(\gamma)\|_{L^{\infty}(\boldsymbol{\pi})}} \int \gamma_{\sharp}|D(f \circ \gamma)| d \boldsymbol{\pi}(\gamma)
$$

on the lattice of measures coincides with $|D f|$. 


\section{References}

[1] Ambrosio, L., Gigli, N. and Savaré, G.: Gradient flows in metric spaces and in the space of probability measures. Second edition. Lectures in Mathematics ETH Zürich, Birkhäuser Verlag, Basel, 2008.

[2] Ambrosio, L., Gigli, N. and Savaré, G.: Calculus and heat flow in metric measure spaces and applications to spaces with Ricci bounds from below. To appear in Invent. Math.

[3] Ambrosio, L., Gigli, N. And Savaré, G.: Metric measure spaces with Riemannian Ricci curvature bounded from below. Available at arXiv:1109.0222v2 [math.DG]

[4] Ambrosio, L. And Dimarino, S.: Weak and relaxed gradients for $B V$ functions on metric measure spaces. To appear. Available at http://cvgmt.sns.it.

[5] BrÉzis, H.: Opérateurs maximaux monotones et semi-groupes de contractions dans les espaces de Hilbert. North-Holland Mathematics Studies 5; Notas de Matemática 50. North-Holland, Amsterdam-London; Americano Elsevier, New York, 1973.

[6] Cheeger, J.: Differentiability of Lipschitz functions on metric measure spaces. Geom. Funct. Anal. 9 (1999), 428-517.

[7] Figalli, A., Gangbo, W. and Yolcu, T.: A variational method for a class of parabolic PDEs. Ann. Sc. Norm. Super. Pisa Cl. Sci. (5) 10 (2011), no. 1, 207-252.

[8] Fuglede, B.: Extremal length and functional completion. Acta Math. 98 (1957), $171-219$.

[9] Gigli, N., Kuwada, K. And Ohta, S.: Heat flow on Alexandrov spaces. Comm. Pure Appl. Math. 66 (2013), no. 3, 307-331.

[10] Gozlan, N., Roberto, C., And Samson, P.: Hamilton-Jacobi equations on metric spaces and transport entropy inequalities. To appear in Rev. Mat. Iberoam.

[11] Hajeasz, P. And Koskela, P.: Sobolev met Poincaré. Mem. Amer. Math. Soc. 145 (2000), x+101 pp.

[12] Heinonen, J.: Nonsmooth calculus. Bull. Amer. Math. Soc. (N.S.) 44 (2007), $163-232$.

[13] Heinonen, J. And Koskela, P.: Quasiconformal maps in metric spaces with controlled geometry. Acta Math. 181 (1998), 1-61.

[14] Heinonen, J. and Koskela, P.: A note on Lipschitz functions, upper gradients, and the Poincaré inequality. New Zealand J. Math. 28 (1999), 37-42.

[15] Koskela, P.: Removable sets for Sobolev spaces. Ark. Mat. 37 (1999), 291-304.

[16] Koskela, P. And MacManus, P.: Quasiconformal mappings and Sobolev spaces. Studia Math. 131 (1998), 1-17.

[17] Kuwada, K.: Duality on gradient estimates and Wasserstein controls. J. Funct. Anal. 258 (2010), 3758-3774.

[18] Lisini, S.: Characterization of absolutely continuous curves in Wasserstein spaces. Calc. Var. Partial Differential Equations 28 (2007), 85-120.

[19] Miranda, M., JR.: Functions of bounded variation on "good" metric spaces. J. Math. Pures Appl. (9) $\mathbf{8 2}$ (2003), 975-1004.

[20] Shanmugalingam, N.: Newtonian spaces: an extension of Sobolev spaces to metric measure spaces. Rev. Mat. Iberoamericana 16 (2000), 243-279. 
[21] Sturm, K.-T.: Generalized Orlicz spaces and Wasserstein distances for convexconcave scale functions. Bull. Sci. Math. 135 (2011), 795-802.

[22] Villani, C.: Optimal transport. Old and new. Grundlehren der Mathematischen Wissenschaften 338, Springer-Verlag, Berlin, 2009.

Received November 29, 2011; revised March 16, 2012.

Luigi Ambrosio: Scuola Normale Superiore, Piazza Cavalieri 7, 56123, Pisa, Italy.

E-mail: 1.ambrosio@sns.it

Nicola Gigli: Laboratoire de Mathématiques J. A. Dieudonné, Université de NiceSophia Antipolis, 06108 Nice, Cedex 02 France.

E-mail: nicola.gigli@unice.fr

Giuseppe Savaré: Dipartimento di Matematica "F. Casorati", Pavia University, Via Ferrata 1, 27100 Pavia, Italy.

E-mail: giuseppe.savare@unipv.it 